\title{
Exploration of elastomeric and polymeric liquid crystals with photothermal actuation: a review.
}

RASTOGI, P., NJUGUNA, J., KANDASUBRAMANIAN, B.

2019 


\section{Journal Pre-proofs}

Exploration of Elastomeric and Polymeric Liquid Crystals with Photothermal actuation: A Review

Prasansha Rastogi, James Njuguna, Balasubramanian Kandasubramanian

PII: S0014-3057(19)30829-8

DOI: https://doi.org/10.1016/j.eurpolymj.2019.109287

Reference: EPJ 109287

To appear in: European Polymer Journal

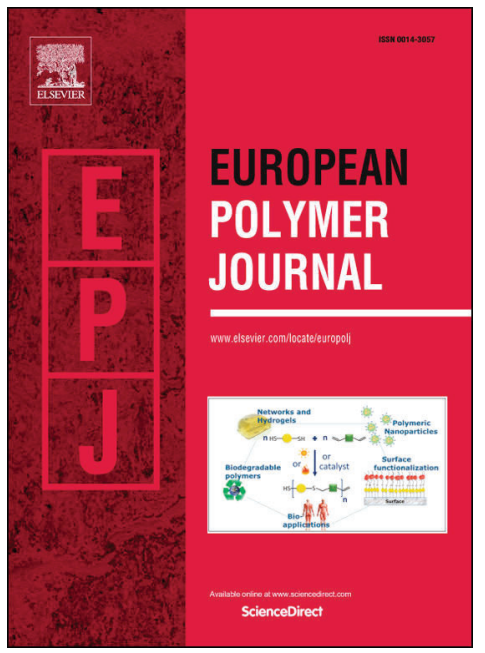

Received Date: $\quad 24$ April 2019

Revised Date: $\quad 14$ June 2019

Accepted Date: $\quad 2$ October 2019

Please cite this article as: Rastogi, P., Njuguna, J., Kandasubramanian, B., Exploration of Elastomeric and Polymeric Liquid Crystals with Photothermal actuation: A Review, European Polymer Journal (2019), doi: https://doi.org/10.1016/j.eurpolymj.2019.109287

This is a PDF file of an article that has undergone enhancements after acceptance, such as the addition of a cover page and metadata, and formatting for readability, but it is not yet the definitive version of record. This version will undergo additional copyediting, typesetting and review before it is published in its final form, but we are providing this version to give early visibility of the article. Please note that, during the production process, errors may be discovered which could affect the content, and all legal disclaimers that apply to the journal pertain.

(C) 2019 Elsevier Ltd. All rights reserved. 


\title{
Exploration of Elastomeric and Polymeric Liquid Crystals with Photothermal actuation:
}

\author{
A Review \\ Prasansha Rastogi ${ }^{\mathrm{a}}$, James Njuguna ${ }^{\mathrm{b}}$, Balasubramanian Kandasubramanian ${ }^{\mathrm{a},{ }^{*}}$ \\ aRapid Prototyping Laboratory, Department of Metallurgical and Materials Engineering, \\ Defence Institute of Advanced Technology (DU), Ministry of Defence, Girinagar, \\ Pune- 411025, India \\ ${ }^{\mathrm{b}}$ Centre for Advanced Engineering Materials, School of Engineering, Robert Gordon \\ University, Riverside East, Aberdeen, AB10 7GJ, United Kingdom \\ *Corresponding Author: Prof. (Dr.) Balasubramanian Kandasubramanian, \\ E-mail: meetkbs@gmail.com
}

\begin{abstract}
Recent research in soft materials is an exhilarating category which has been transcending boundaries for variety of functional applications. This category also stems liquid crystals whose stimuli-responsive feature has fantasized researchers for application arrays in actuators and biomedical. Liquid crystals evince dual characteristics of liquid and solids empowering them to reversibly transit on external actuation. The after-effect of irradiating photons on liquid crystals (LC) facilitate outlying functioning and are engineered with gold nanorods, dyes, graphene and carbon nanotubes among others which greets to incoming stimulus and participates in transference of light energy to perceivable transformation through heat drive. This is progressively explored in medical domain for drug delivery, tissue engineering, cancer treatment, and other disciplines of medicine and bio-mimicking. Additionally, photothermal trigger equips localized punctilious treatment outshining diffusion assisted heating and enables spot treatment. However, LC utilization is burgeoning towards 3D printing,
\end{abstract}


characterizing it as 4D Printing. Present review framework probes LC and its photothermal actuation chemistry in the medical domain. Furthermore, it reflects on LC potential in smart manufacturing in 3D/4D printing, its challenges (limited concentration of filler, its miscibility, and actuation cycle fatigue) and future likelihood.

Keywords- Liquid Crystals; Photothermal; Biomedical; 3D/4D Printing; Gold Nanorods; Azobenzene

\section{Graphical Abstract}

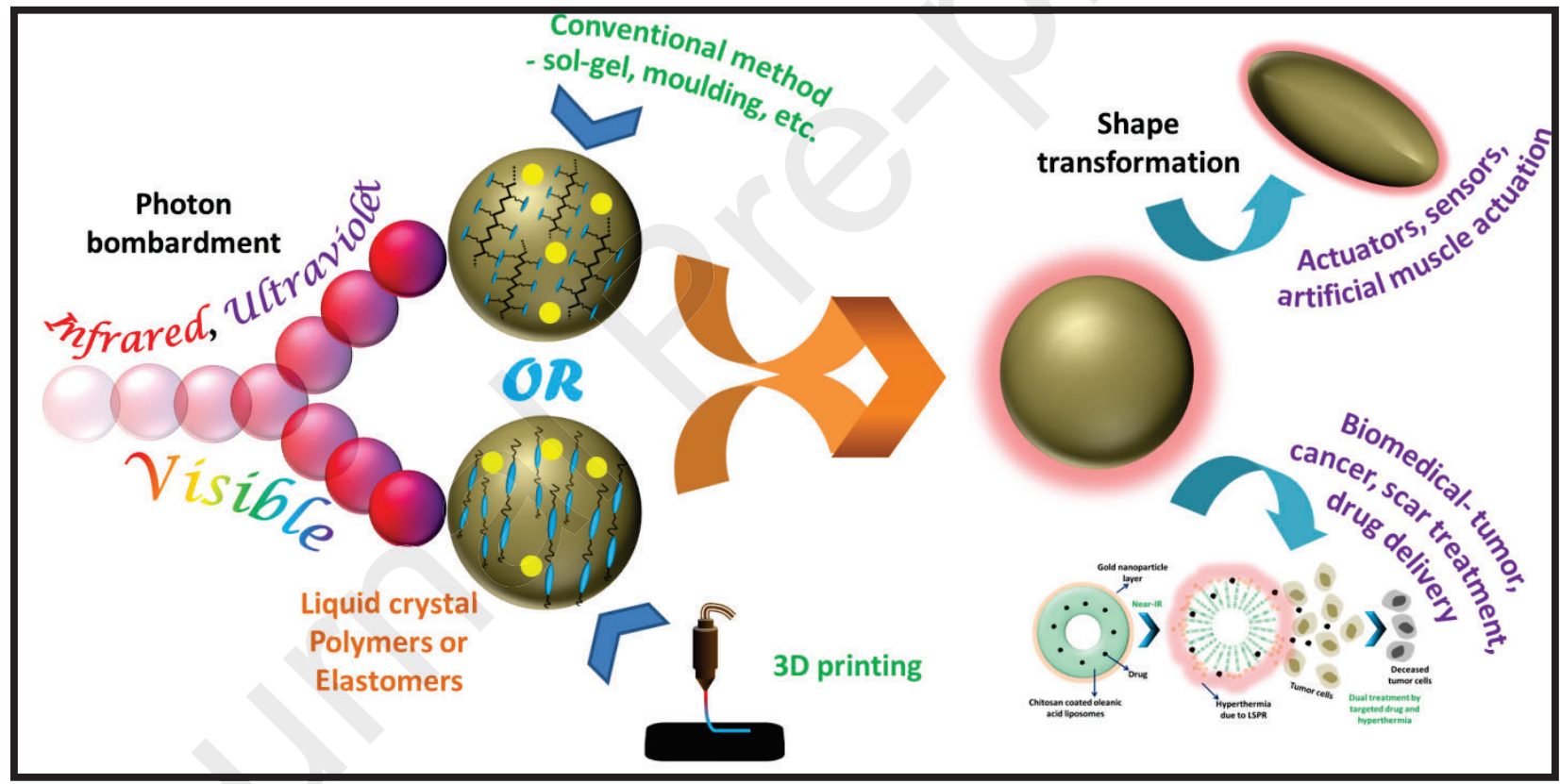

\section{Introduction}

The architectural design of nature is mysterious and magical; whose imitation in research applications is an endeavor such as in soft materials like Liquid Crystals (LC). Market Analysis envisioned LC's global exploitation to outstretch to $\sim 80$ kilotons by the year 2020 
from 42.8 kilotons in 2012 and 70 kilotons in 2018 acquiring 1.2 billion USD market share in forthcoming couple of years (2020) with 6\% growth rate. Among various applications of LC's i.e. medical, automobiles, machinery, and other consumer goods, electrical-electronic (missile electronics, sensors, weapons, LEDs, switches, LCDs etc.) exploitations are ruling the dominion (major pie) and continue to ascend in succeeding years. However, analysis has also shown boost in utilization of LC in medical on account of its ordering-disordering property. Regionally, Asia-Pacific and especially, China will continue leading LC market ( $>45 \%)$ along with an expectednoteworthy development in Indian and Indonesian regions [1,2]. Vision of LC market is huge and will witness further exploration in other fields (except electricalelectronics) like automotive, medical, machinery etc. Thus, witnessing their impactful research applications and performances, scientists are increasingly dedicating their research on further exploration of LC in variegated domains.

Friedrich Reinitizer in 1888 discovered the presence of mesophase and dual melting transitions in material while researching on carrot derived cholesterol, which was later characterized by Otto Lehman on optical microscopy marking the origin of LC study [3]. LCs have two contrasting flavors (first, solid's orderly arrangement of molecules for anisotropy and second, liquid's non-orderly isotropy \& fluidity) whose fusion has engineered the material to attain reversible shape change/memory and optical characteristic [4]. Thus, LC embodying anisotropy represents intermediate state of crystalline solid (characterized at below T-melting) and liquid (above T-melting) in which configuration evidenced destroyed positional order in accordance with later while preserved orientation corresponding to former (Figure 1 (a)), thus witnessing combined properties from both phases. This orientational order is memorized by mesogens when bombarded by external energy to increase entropy resulting is shape change 
(transition to different phase) and with stimuli retraction, it reconquered parent configuration.

Principal structure of mesogen-containing LC are delineated in Figure 1 (b) with long and stiff, core-derived circular or amphiphilic design (explained further in this section) [5].

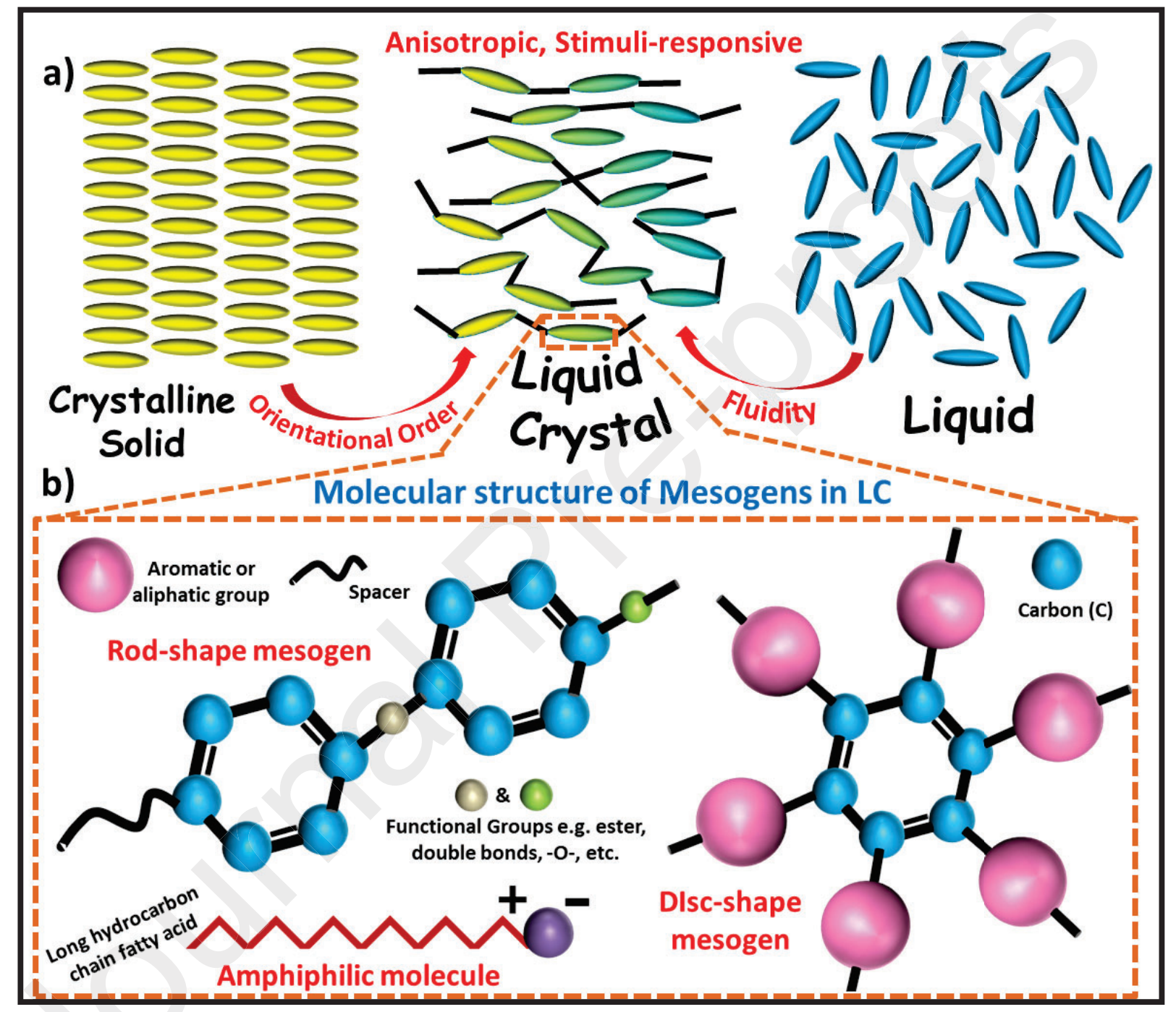

Figure 1. a) Derivation of liquid crystals as an optimized state of liquid and crystalline solid in order and properties. Gradient color in LC represents influence of both phases. b) Depicts mesogenic conformation in LC i.e. rod-, disc-shaped and amphiphilic-type (major in lyotropic LC).

Above-mentioned characteristics are endowed by organizing mesogens around the director axis which is portrayed in Figure 2 along with other classifications driven by different 
rationales. In relation to Figure 2, classification of LC is predicted by director orientation i.e. perpendicular, angular, spiral, or multiple directionalities defining nematic, smectic, cholesteric, and isotropic structure respectively (smectic can have perpendicular or angular director axis but mesogens are in a line as shown in Figure 2). Nematic LC class evinces long-range orientational order in mesogens parallel to director axis but mobility freedom along same direction implies short-range order in position thus accredits to anisotropy in electrical, optical, viscosity and magnetic properties. In contrast to above, smectic phases showcase long-range order in position conjointly with orientation i.e. biaxial order. Though these phases manifest parallel relationship with director axis but the later may display an angular tilt with respect to vertical axis engendering smectic A (director axis parallel to vertical normal axis) or $\mathrm{C}$ phase (director axis at an angle to vertical normal axis determined by temperature). Other than these common types include B, E, F, etc., relying on mesogenic or crystal structure arrangement like hexagonal (B-type) and orthorhombic (E-type). However, next classification i.e. cholesteric, also known as chiral nematic phase, has director axis in which mesogens continuously changes directions in form of helix. Pitch of the helix is defined when mesogens have accomplished rotation of $360^{\circ}$ either from right-handed or left-handed direction governed by conformation which enkindles iridescent colors (due to fine-tuning in helical pitch of LC phase). Isotropic exemplifies the randomized orientation of mesogens with no directionalization of director axis and appears when energy transference from external stimuli exceeds a threshold. [4,6,7]. Abovesaid structure can acclimatized to disordered isotropic phase from ordered assisted by an external trigger owing to differential conformational entropy. For instance, nematic LCs are ordered having worm-alike configuration of mesogens (free volume low) and energy incidence induces stress which 
variegates entropy transforming to coil-alike (more free volume for adjusting random aligned mesogen) contracted structure contributing shape transition [5,8]. Further classifications rely on the location of mesogens (main or side chain), primary processing (thermotropic and lyotropic), and extent of bonding (mild or extensive which renders LC-Polymer, LCElastomer, and LC-Network whose comparison has been shown in Table 1) [6]. In mainchain, mesogens forms integral part of LC polymeric backbone while in side-chains mesogens exist as branches that are protruding out from main backbone. The later also facilitates covalent attachment to backbone without disruption rendering enhanced solubility and hence, enables substantial additions e.g. dyes to form stimuli-responsive LC [9]. In addition, mesogenic units present on main chain or side branch (further grouped to end-on and side-on) render modulations in characteristics i.e. main-chain, owing to stronger bonding on either side, delivers enhancement in mechanical properties when compared to side-chain LC $[3,10]$. However, similar properties can also be achieved to an extent with bulky side groups on mainchain which contributes to impediment-directed stretching. Temperature plays a characteristic part in thermotropic LC i.e. mesophase alters when ordered solid is heated or disordered liquid is cooled. Corresponding to LC thermodynamic stability, enantiotropic mesophase arises during both heating and cooling while monotropic arises only during heating. Lyotropic LC phase, on contrary are influence by concentration of solute which are amphiphilic in nature as well as temperature. These molecules are constituents present in soap/detergent, DNA, polysaccharides, cosmetic, polypeptides, tobacco mosaic virus etc. in various food and biomedical domains (drug delivery) [6,7,11]. The phase can be sub-classified into chromonic phase corresponding to molecular structure as it habitats ionic group at outer and hydrophobic group at inner [12]. Later classification (LC-polymers, elastomers, networks) is an archetype 
of bonding or crosslinking between molecular chains of LC, lighter the linking feeble is the chance for stimuli-responsiveness. Elastomers delineate mild crosslinking which empowers sufficient stiffness to bestow fixation to temporary shape and meantime, flexibility to chain movement for recovering parent structure under external stimulations. In addition, crosslinking not only mechanically but also orientationally favors structure in nematic phase thus ensuring transition temperature shift to positive side and further amelioration was witnessed with heterogeneous LC formulaion. Mechanism follows shuttling of LC molecular chains between orderly and disordered or isotropic state at a transition temperature [13-15]. Molecular structure of mesogenic units in LC depicts variegated geometry i.e. calamitic (organic molecules depicts rod alike arrangement (length $>>$ diameter) with rigid link type structure conjoined with flexible hydrocarbon tail), discotic (resemblance with disc shape having rigid core of benzene ring, truxene, triphenylene, etc. surrounded by flexible tails), and slightly bent banana configuration with adjacent spacer molecules (Figure 2) $[6,16]$. Spacers are flexible molecular chains parting mesogens or/and acts as linker to the backbone chain in LC which aids their respective motion when signaled by a particular stimulus. Carbon number in spacer also regulates interactions (steric, $\pi-\pi$, or decoupling effect) and phases (columnar or hexagonal) as a function of governing properties in side-chain LC (Figure 2) [17]. These mesogens of LC constitute main chain of thermoplastic or thermosetting units e.g. siloxanes (Si-O linkage), epoxy, ester, Vectran, amides, acrylates (owing to their photo-polymerization e.g. RM82, RM23, RM257 etc.), polyvinyl pyridine, polyacrylic acid, lipids, etc. [18-22] along with some additives that render it responsible to direct/indirect stimuli.

Other phases includes columnar phases (further classified into hexagonal and rectangular based on arrangement of mesogens), chiral nematic, chiral smectic, blue phases, twist grain 
boundary phases, homeotropic and planar (based on mesogenic orientation) and ferroelectric LC [7]. Blue phase exists for short temperature range between nematic LC possessing chirality (directionalization) and randomized isotropic LC which also restricts its wide utilization industrially. They further exists in blue phase I, II, and III depending on crystal structure defined by simple-, body-centered cubic crystal structure and amorphous respectively (later also interprets fog-type resemblance) with influential utilization in photonics and fast displays. In addition, chirality manifests directionality but symmetry deficiency for e.g. in smectic $\mathrm{C}$ which induces polarity and hence, initiates stimuliresponsiveness (e.g. electric field which determine mesogens orientation, light etc. enables its employment in switches, motors etc.) to formulated LC. Moreover, in contrast to blue phase, twist grain boundary phase (TGBP) prevails at the border region of chiral smectic C phase transforming to isotropic phase. Conflict between chirality and positional order of smectic phase is compromised when layers clubs together in form of twist grain boundary and each boundary displays rotation in helix form which also resembles screw dislocation. These can further be grouped to TGBP- A, C and C-chiral based on smectic phase present for transition [7,23-26]. Ferroelectric LC owing to pre-occupied polarization (groups with dipole moment e.g. carbonyl) and chiral structure contributes to quick alignment of mesogens in direction of externally electric field therefore, contributing to swift reaction in shorter time. This switching of mesogens are necessitated in optics and electronics (as polarization in electric field can induce visible optical change) tuned by pitch of chiral smectic phases. Nevertheless, LC can be devised photo-actuable when integrated with photo-responsive molecules e.g. azobenzene. These molecules tends to dwindle coercive force (generated with $10 \mathrm{~V}$ electric field) after trans (stable) $\rightarrow$ cis (unstable) transformation at $360 \mathrm{~nm}$ irradiation causing speedy microsec 
mesogenic flips for storing information. However, longer wavelengths of $\sim>450 \mathrm{~nm}$ can be employed to sweep the stored image clear and for later rewriting $[27,28]$.

Aforementioned classified phases cease to exist in solitary i.e. thermotropic LC-Elastomer having nematic phase with rod-shaped mesogens can be accompanied by isotropic phase when transited beyond transformation temperature. As an instance, Zou et al. demonstrated triphenylene derived NPT6 and HAT6 LC (differed by ester group attached) possessing disclike mesogens arranged in a hexagonal columnar phase for sensors and organic transistors. Authors further reported that NPT6 and HAT6 structures had supported homeotropic and planar alignment respectively in which current density ameliorated 4 times in later while former remained unchanged at $80 \mathrm{~V}$ (owing to faster electron transport ascribed to parallel mesogens orientation in HAT6) [29]. Authors also doped alkene motors (UV light sensitivity) in LC (HAT8) to witness UV-guided switches and memory devices relying on electricity. These switches displayed intensified conductivity with $4 \mathrm{~mW} / \mathrm{cm} 2 \mathrm{UV}$ but it ceased with $>16$ $\mathrm{W} / \mathrm{cm}^{2} \mathrm{UV}$ ascribable to disturbed columnar alignment. In addition, LC evinced dual property of self-healing and memory device $\left(>16 \mathrm{~mW} / \mathrm{cm}^{2}\right)$ in which both can be activated by UV illumination $\left(200 \mathrm{~mW} / \mathrm{cm}^{2}\right)$ followed by $\sim 70^{\circ} \mathrm{C}$ heating-cooling (also employed for erasing data) [25]. Trček et al. had observed TGBP transiting amidst smectic A phase and cholesteric phase when gold nanoparticles with dimensions in range $\sim 10 \mathrm{~nm}$ was added in chiral CE8 LC. The phase was validated by various characterization tools and additionally, it had also caused shifting in surface plasmon resonance (explained in Section 2) peak to $\sim 650 \mathrm{~nm}$ with extra shoulder at $870 \mathrm{~nm}$ confirming its (TGBP) clustered presence [26]. However, increasing dimensions can lead to stabilization of blue phase as demonstrated by Lavrič et al. with same LC but laponoite clay nanoplalets in 2 mass $\%$ ( $25 \mathrm{~nm}$ by $1 \mathrm{~nm}$ dimension) and graphene in 0.1 
mass \% with potential applicability in electro-optical devices. Formulated LCs enhance stability to $6 \mathrm{~K}$ and $7.5 \mathrm{~K}$ respectively for clay and graphene (when compared to neat CE8) with both promoting formation of blue phase-I over others owing to considerable surface areas of both reinforcements [23,24].

Table 1.Comparison of different LC.

\begin{tabular}{|c|c|c|c|c|}
\hline Properties & LC-Polymer & LC-Elastomer & LC-Network & References \\
\hline Crosslinking & No & Mild & Extensive & \multirow{6}{*}{$\begin{array}{c}{[19,20,30-} \\
34]\end{array}$} \\
\hline $\begin{array}{l}\text { Change in } \\
\text { order after } \\
\text { stimulus }\end{array}$ & No change & $\begin{array}{l}\text { Slight change } \\
\text { (around 5\%) }\end{array}$ & $\begin{array}{l}\text { Substantial change } \\
\text { (90\% or above) }\end{array}$ & \\
\hline $\begin{array}{l}\text { Shape } \\
\text { memory }\end{array}$ & $\begin{array}{l}\text { No (no driving force } \\
\text { i.e. crosslinks) }\end{array}$ & $\begin{array}{c}\text { Exhibit shape } \\
\text { memory }\end{array}$ & $\begin{array}{c}\text { Exhibit shape } \\
\text { memory }\end{array}$ & \\
\hline $\begin{array}{c}\text { Glass } \\
\text { transition } \mathrm{T}, \\
\mathbf{T g}\end{array}$ & $\begin{array}{l}\text { Very high } \mathrm{Tg} \text { (above } \\
\left.200^{\circ} \mathrm{C}\right)\end{array}$ & $\begin{array}{l}\text { Below room } \mathrm{T} \\
\text { i.e. } 25^{\circ} \mathrm{C}\end{array}$ & $\begin{array}{l}\text { In range of } 60^{\circ} \mathrm{C} \text { to } \\
\qquad 100^{\circ} \mathrm{C}\end{array}$ & \\
\hline $\begin{array}{l}\text { Elastic } \\
\text { modulus }\end{array}$ & $\operatorname{High}(>2 \mathrm{GPa})$ & $\begin{array}{l}\text { Low (below } \\
100 \mathrm{MPa})\end{array}$ & $\begin{array}{l}\text { Medium (in between } \\
\text { LC-Polymer\& LC- } \\
\text { Elastomer) }\end{array}$ & \\
\hline Examples & $\begin{array}{l}\text { Vectra (copolyester } \\
\text { of p-hydroxy } \\
\text { benzoate in } \\
\text { formulation with 2- } \\
\text { hydroxy -6- } \\
\text { naphthoic acid } \\
\text { having aromatic } \\
\text { structure), Kevlar } \\
\text { (poly-paraphenylene } \\
\text { terephthalamide) }\end{array}$ & $\begin{array}{l}\text { Siloxane main- } \\
\text { chain with vinyl } \\
\text { mesogen }\end{array}$ & $\begin{array}{c}\text { Polymerization of } \\
\text { RM23 (4- } \\
\text { cyanophenyl-4'-(6- } \\
\text { acryloyloxyhexylox } \\
\text { y)benzoate) with } \\
\text { RM82 (1,4-bis-[4- } \\
\text { (6- } \\
\text { acryloyloxyhexylox } \\
\text { y)benzoyloxy]-2- } \\
\text { methylbenzene) }\end{array}$ & \\
\hline
\end{tabular}




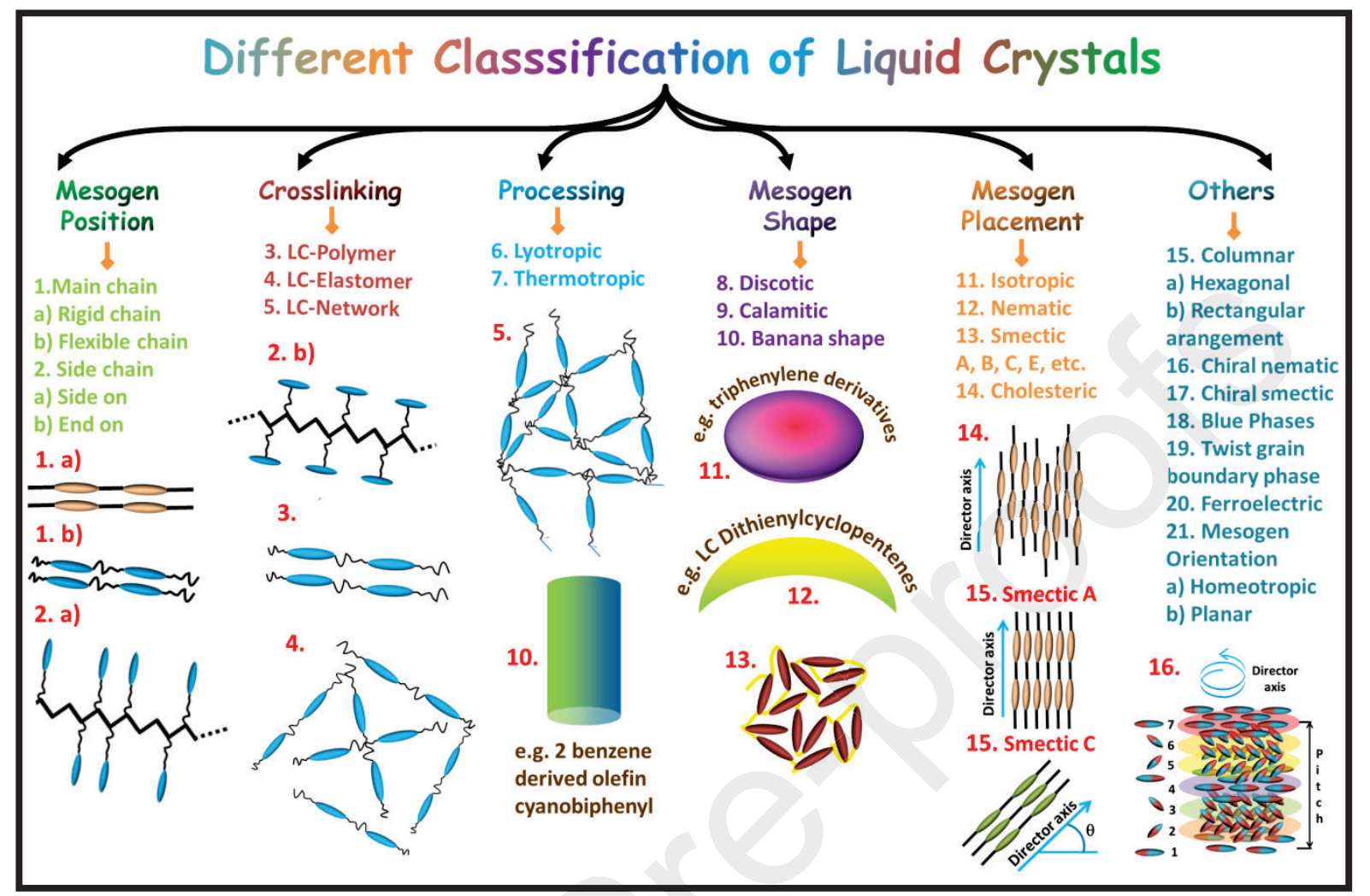

Figure 2. Different classifications of liquid crystals.

Tao et al. had reported color variation under UV actuation by varying proportion of hydrogenbonding acceptor (4,4'-dibutanoxy tetraphenyleethylene)-1-pyridine (BTPEP) $)_{y}$ in luminescent LCP. They observed inverse proportionality of BTPEP with Tg due to plasticization while proportional relationship advocating LC structure in formulations. They further demonstrated that increasing amount (i.e. y) of BTPEP not only caused transition in phase from smectic-A $(y=1-0.8)$ to columnar nematic $(y=0.6-0.05)$ to amorphous $(y<0.05)$ but also light emission red-shifted to $551 \mathrm{~nm}$ at $\mathrm{y}=0.025$ from $510 \mathrm{~nm}$ at $\mathrm{y}=1$ [35]. Boothby et al. had amalgamated hydrophilicity and LC characteristics in layered format to witness simple as well as complex stimulations with water and heat relying on mesogen orientation. Authors designed bilayers with RM82 LC and poly(ethylene glycol)diacrylate (PEGDA) based hydrophilic polymer. Tailoring mesogenic director axis alignment in LC layer i.e. varying from $0^{\circ}$ to $90^{\circ}$ caused 
pattern to anisotropically display folding at $0^{\circ}$, low pitch helix and high pitch helix at intermediate angles with finally spiral shape at $90^{\circ}$ under stimulus (Figure 3). More intricacy in designing can be achieved with multiple orientation directions of mesogens in a single entity as shown in Figure 4 [36]. Nevertheless, mechanical properties of RM82 can be customized by at least 2-folds (storage modulus) after crystallization in disorderly isotropic and orientational-ordered nematic phase as studied by Kim et al. Authors discovered that alignment and crystallization has ameliorated strain at failure $(\sim 1396 \%$ in perpendicular), toughness $(\sim 40 \mathrm{MPa}$ in parallel), and ultimate tensile strength $(\sim 78 \mathrm{MPa}$ in parallel) for isotropic-LC over $668 \%, \sim 16 \mathrm{MPa}$, and $\sim 48 \mathrm{MPa}$ respectively but declined modulus of $\sim 355$ $\mathrm{MPa}$ ( 439 MPa for isotropic) for nematic-LC. In contrast, owing to crystallization both structure prevented changes in Tg and configuration (shape) prior to melting. Moreover, prepared LC exhibited $\sim 18$-folds $\left(730 \mathrm{KJ} / \mathrm{m}^{3}\right)$ enhancement in work capacity under actuation than muscles of mammals $\left(40 \mathrm{KJ} / \mathrm{m}^{3}\right)$ [37]. Similar to above-mentioned research for multiple orientations in a single entity but to a complete depth, straining and selective UV exposure could weave mesogens in mono- or multi-domain alignment to desired depth. This fabrication technique assists in tailoring entities by merely varying the incident light and its time of illumination for crosslinking. Area, depth, distribution, pattern, and location of exposure engender discrepancy in induced force due to crosslinking which acts as driving force for complicated stimulated framework. As an instance, as depth of incident UV light (320 nm) on cinnamyl containing LC increases (initiates deeper crosslinking), thermal actuation advances design from bending to buckling to rolling (Figure 5). Similarly, thermal exposure on aligned (other than $90^{\circ}$ or $0^{\circ}$ with horizontal/verical) mesogens tailors helix and distributed UVcrosslinking bestows structure its wrinkling or wave-like design (Figure 5) [38]. 


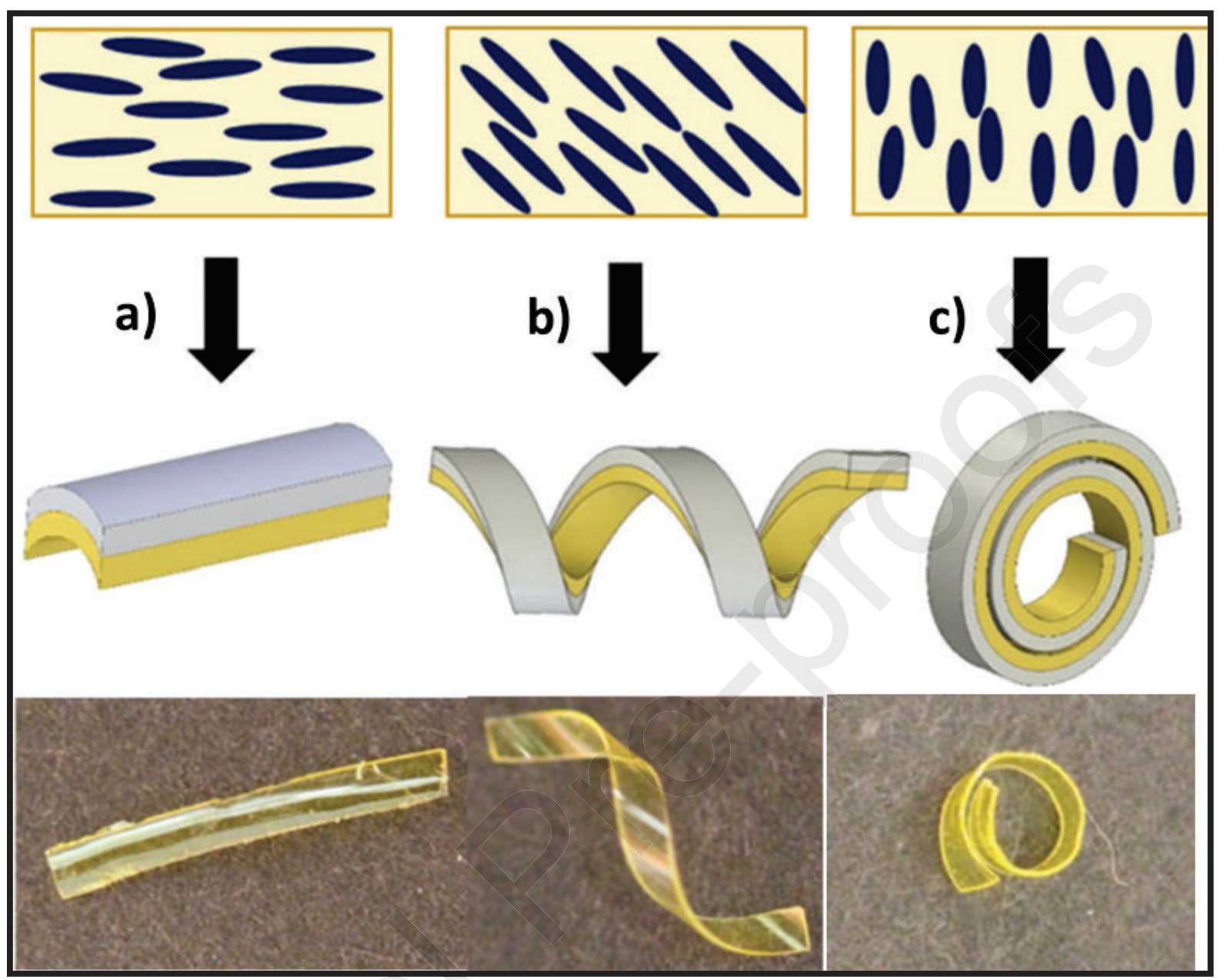

Figure 3. Illustration of mesogen alignment on the derived shape after stimulus on bilayer PEGDA and RM82 which endows design flexibility from longitudinal bent-like structure to spiral [36]. 


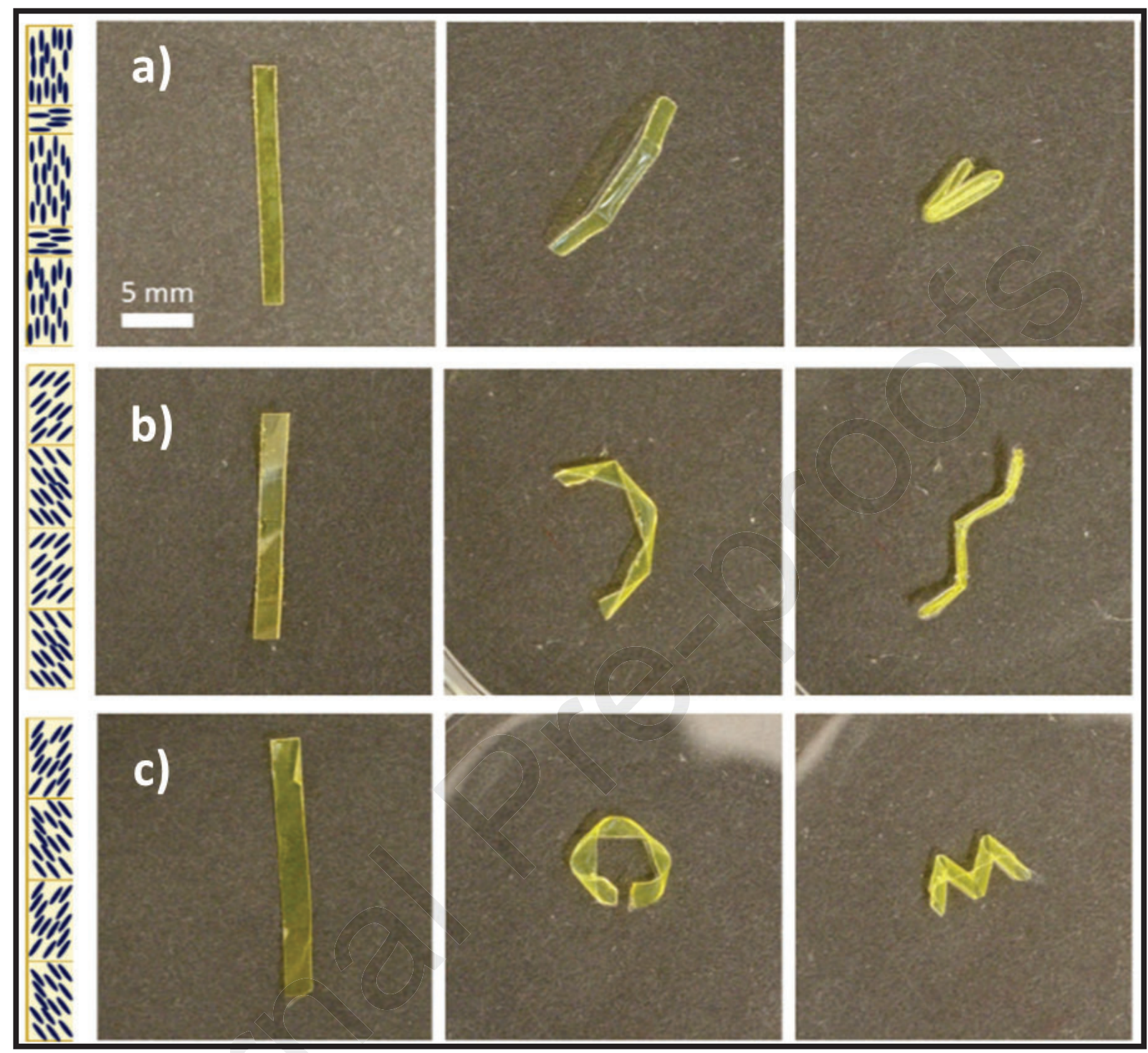

Figure 4. Demonstrates complex tailoring of actuation by different arrangement of mesogens while processing [36]. 


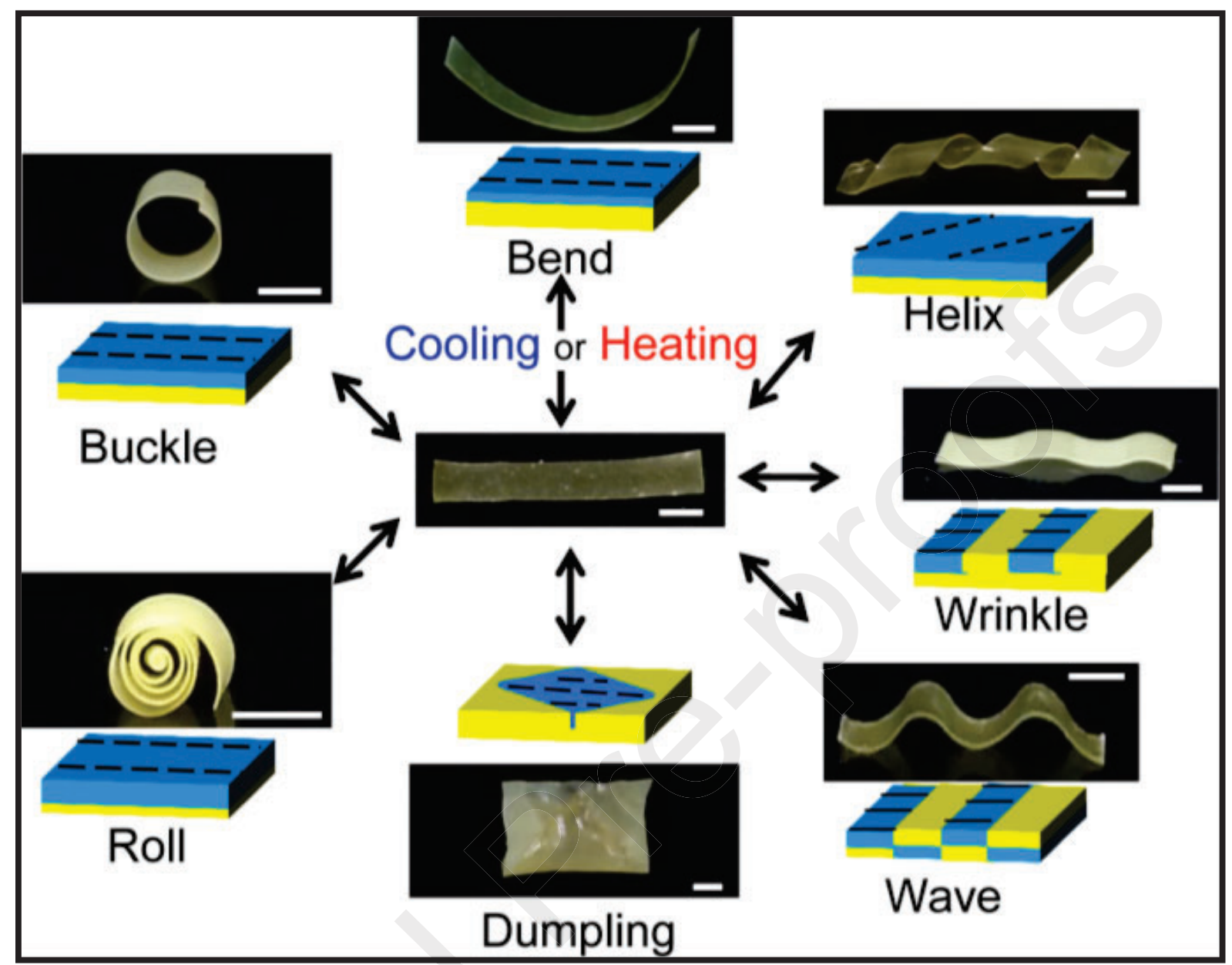

Figure 5. Elucidates relationship of UV exposure bestowing crosslinking on depth and pattern which meanwhile guides actuation pattern i.e. helix, buckling, wrinkling, rolling, dumpling, bending etc. [38].

Additives attach themselves with LC chains (generate network-type construction) and serve to improve its functioning e.g. Thapa et al. investigated graphene oxide (GO) embodiment in hybrid LC matrix which bestowed photothermal proficiency for apoptosis of cancerous cells in near-infrared (near-IR)and simultaneously, servicing for drug delivery [39]. This responsive character of LC was amplified by GO which can also be obtained by other reinforcements i.e. carbon nanotube $(\mathrm{CNT})$, graphene or reduced-GO, polydopamine, gold $(\mathrm{Au})$, silver $(\mathrm{Ag})$, azobenzene, pyrene, aniline, anthracene, etc. for miscellaneous triggering sources with an endapplications in actuator, medical, sensors, etc. [18,22,40-42]. Incorporating these agents, 
responsiveness (actuation \& recovery back to original) can be accomplished by direct heat, $\mathrm{pH}$, and indirect heat as in IR, current, Ultravoilet (UV), magnetic field, Visible (Vis) etc. [21]. These triggers interact with building blocks of material and deliver their energy to them which engenders structural or functional alteration that is perceivable at macroscale (details in subsequent sections) [43-45]. This transference of energy generates strains or residual stresses which are recovery guidance for shape reversal [46]. This driving force compels entity to encounter 2-D change in form of contraction/expansion or 3-D movement typifying bending which characterize application like actuators, sensors, in microfluidics, and other engineering applications. In addition to aforesaid, LC (being a polymer, elastomer or a network) renders practicability by endowing weight advantage, operation compliance, processing efficacy, flexibility, ability to fine-tune properties by reinforcements and cost effectiveness. Furthermore, these materials displays expeditious response (prerequisiste for such applications), mesogen alignment in electric fields, prominent deformation, low-stress transition- large strain thus likelihood for room-temperature workability (thermostricitve behavior), anisotropy in properties, shape memory (recovery and fixity) for multiple cycles, short space link connecting mesogen and backbone, etc. These materials greet thermal energy (LC $\leftrightarrow$ isotropic transition) but reinforcements proffer expansion in stimulations to light (cistrans by azobenzene, photothermal by near-IR in CNT, gold, silver etc.), electrical or magnetic stimulation elide on additives nature. Correspondingly, smaller spacers strengthen bonding and interaction among mesogens-LC backbone which not only confers requisite anisotropy but also weave framework for mimic muscles actuators $[5,8,15,47,48]$. As an illustration, Thomsen et al. reported benxoate derived elastomers with acrylate-based crosslinker upto $45 \%$ geometrical change with modulus $\sim 0.88 \mathrm{MPa}$ by changing monomer for 
LC (2 different benzoate). Authors also studied recovery force for actuation, $270 \mathrm{MPa}$, which was in direct relation with conformational entropic change while reversibly transiting from nematic to isotropic thus, posing a resemblance with natural muscle for artificial actuators [8].

Diverse characterization tools assist for evidencing the presence/absence of any functionality/process/dimension/etc. Namely, creep and dilatometry which are experiments to evaluate the structural adaptation to increasing stress, T, \& time simultaneously determining the elastic $\rightarrow$ plastic $\rightarrow$ melt state along with many other functions. Apart from other contemporary characterizations, like polarized optical microscopy (POM) identifies the director axis of mesogens in LC and thereby, chain alignment from perceivable birefringence. Orientation order of mesogens and thereby chain controls anisotropy which is bestowed in 3D printing by shear-driven extrusion stress from nozzle walls and printing speed. These parameters in addition, also monitors stretch-ability of mesophase (while dispensing from nozzle) in line with printing (which also guides relaxation followed by transformation) [49]. Thus, 3D additive technique empowers the scientists to write or dispense complexity-ridden shapes and their actuation e.g. meanders, spirals, mesh etc. [50]. 3D printing coupled with LC (displaying shape memory behavior) engenders the term 4D printing coined by Skylar Tibbits in which he explained about the practical reframing of residual stress into stimuliresponsiveness of polymers [51]. Scopus and Web of Science analysis revealed the scope of LC 3D printing (i.e. 4D Printing) as a potential future manufacturing technique owing to its inherited advantages which can be perceived with its limited literature availability. The literature analysis (Scopus and Web of Science) for 4D printing, shows a limited availability of documents $(<300)$. However, the literature trend also shows that, the scientific community has started exploration in 4D printing research domains at a burgeoning rate since 2013 
(Figure 6). Since LC inherits transform-ability, therefore, the alliance has unlocked multiple architectural designs in engineering and medical with complex 2-way stimulation. Further, an addition of biodegradability and self-healing (in UV or Vis at low temperature (T)) in LCelastomers has augmented the scope in biomedical precision (tissue engineering- scaffolds etc.) applications $[52,53]$. LC's complex non-intervened actuation by light coupled with heatguided transformation i.e. photothermal analysis is depicted in Figure 7 as per Web of Science analysis.

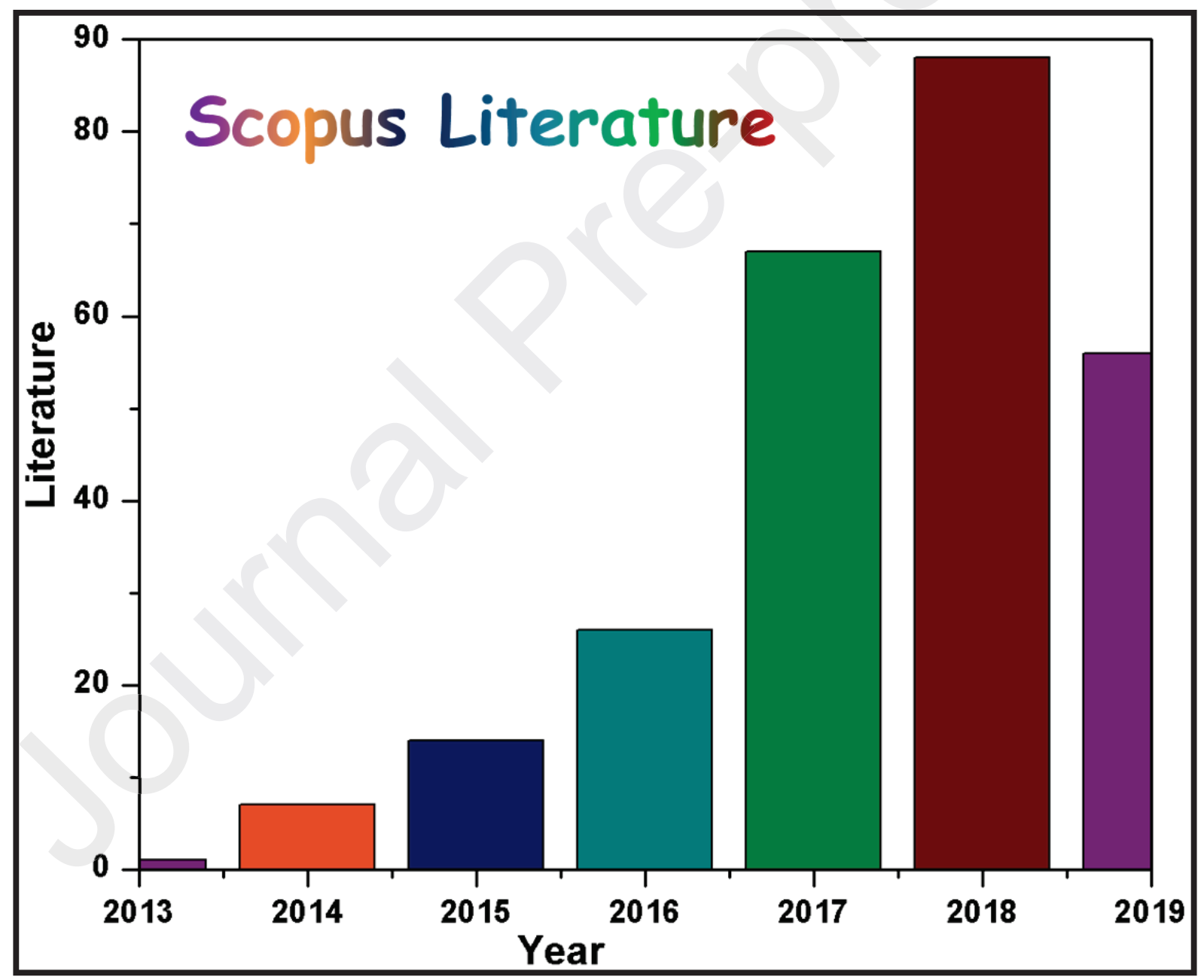

Figure 6. Scopus literature survey data on 4D printing since its introduction in 2013. 


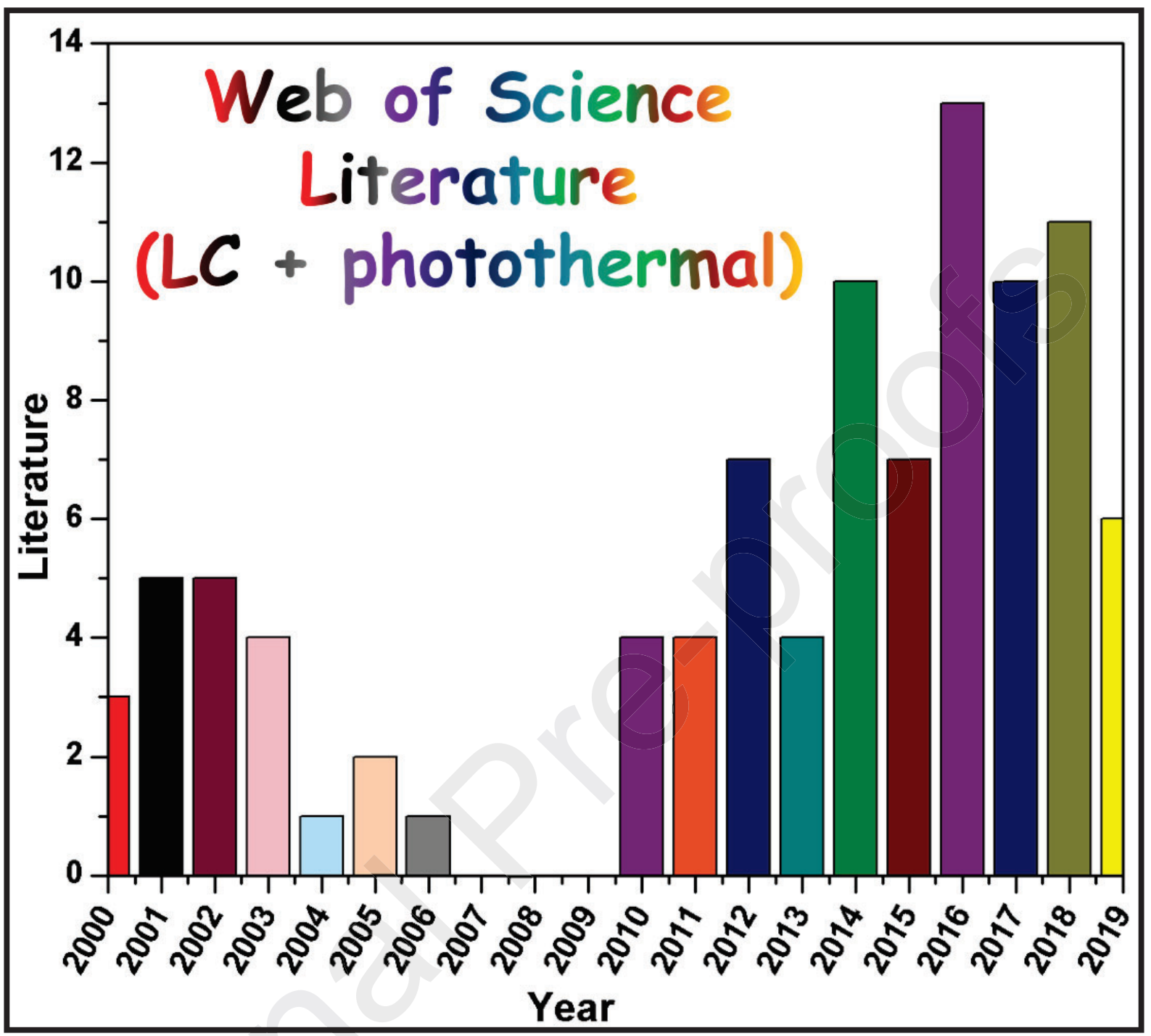

Figure 7. Web of Science literature analysis on LC being stimulated by photothermal effect which defines a wide scope for in-depth research.

Present review pivots photothermal influence in LC, which being a harmless source, accomplish its functioning as a solo army triggering perceptible macro-alteration remotely. The open literature on photothermal, LCs and 3D printing individually are numerous but bracketing LC with photothermal and driving it towards biomedical applications while taking in account 3D/4D Printing is still at its infancy. With the advent of 4D Printing, the research is escalating globally for multifarious applications in actuating, sensing, soft robotics, etc. with biomedical being the prime. Accounting the fact, review discusses the chemistry of additives 
(e.g. dyes, Au, etc.) for photothermal actuation and the resultant outcomes concerning stimulus, concentration (conc.), temperature rise, self-healing and other thermal \& mechanical aspect in LC. Furthermore, it also defines the potential future of 3D/4D printing in LC which can be stimulatedwith light having capability to generate photothermal effect, also unveiled in published works on Scopus and Web of Science. LC, being a multifunctional entity, proffers for high-end application and associating it with additive manufacturing further aids in simplifying and understanding of remote complex implementations.

\section{Chemistry of additives in photothermal actuation}

Absorption of photons which assist in consequential transitions are served in equity for both photo-responsive and photothermal i.e. mechanism of light assimilation in both are same. Macro-scale accompanied transformations are the collections of numerous micro-/nano-scale changes triggered by photons which otherwise are constrained due to its particles/molecules size, shape, concentration (conc.) etc. dependency [54].

Azobenzene, an organic molecule photo-isomerizes around "-N=N-" bond which reversibly adapts to trans-cis planar-bent conformation on exposure to UV/Vis. The chemistry behind is rather complicated, but basically governed by $\mathrm{n}, \pi$, and $\pi^{*}$ bonding states in thermodynamically stable trans-azobenzeneand less stable cis-azobenzene [4], basics behind light response in the molecule is defined as-

a) Absorption: it is the photonic energization of electrons with light, activating $n / \pi$ electrons to $\pi^{*}$ state which characterizes cis-trans actuation respectively (extent of $\pi \rightarrow \pi^{*}>$ $\mathrm{n} \rightarrow \pi^{*}$, therefore trans absorbs wavelength at high energy i.e. $320 \mathrm{~nm}$ while in cis, out-of- 
plane bent deformation is carried out at low energy, i.e. $430 \mathrm{~nm}$ for azobenzene). However, this wavelength can be attuned to visible range by various substitution e.g. amino-benzenes, 4methylamino-4'-nitro-azobenzene etc. produces red-shift. Quantitative efficiency of cis product (i.e. after absorption) is augmented with illuminated time without subsequent observation in the phosphorescence/radiative decay/emission after transition [55-57].

b) After-effect: Trans signal diminishes with time contributing complete cis-transition and conformation is driven when excited trans matches the cis state producing resonance in wavelength therefore, driving geometrical bending. As an after-effect, dislocated cislone pair electrons of nitrogen $(\mathrm{N})$ modulate dipole momentand strain the structure, which furthermore enhances with crosslinks. To revert back the structure to trans (strain-free planar state), enthalpy of activation, $\sim 96 \mathrm{~kJ} / \mathrm{mol}$ with thermal/Vis $(400-800 \mathrm{~nm})$ energization is indispensable for piloting 2-way reversibility. Lower energy for reversion (back to stable trans) was attributed to in-built strain developed due to unstable cis structure (Figure 8) [5557]. 


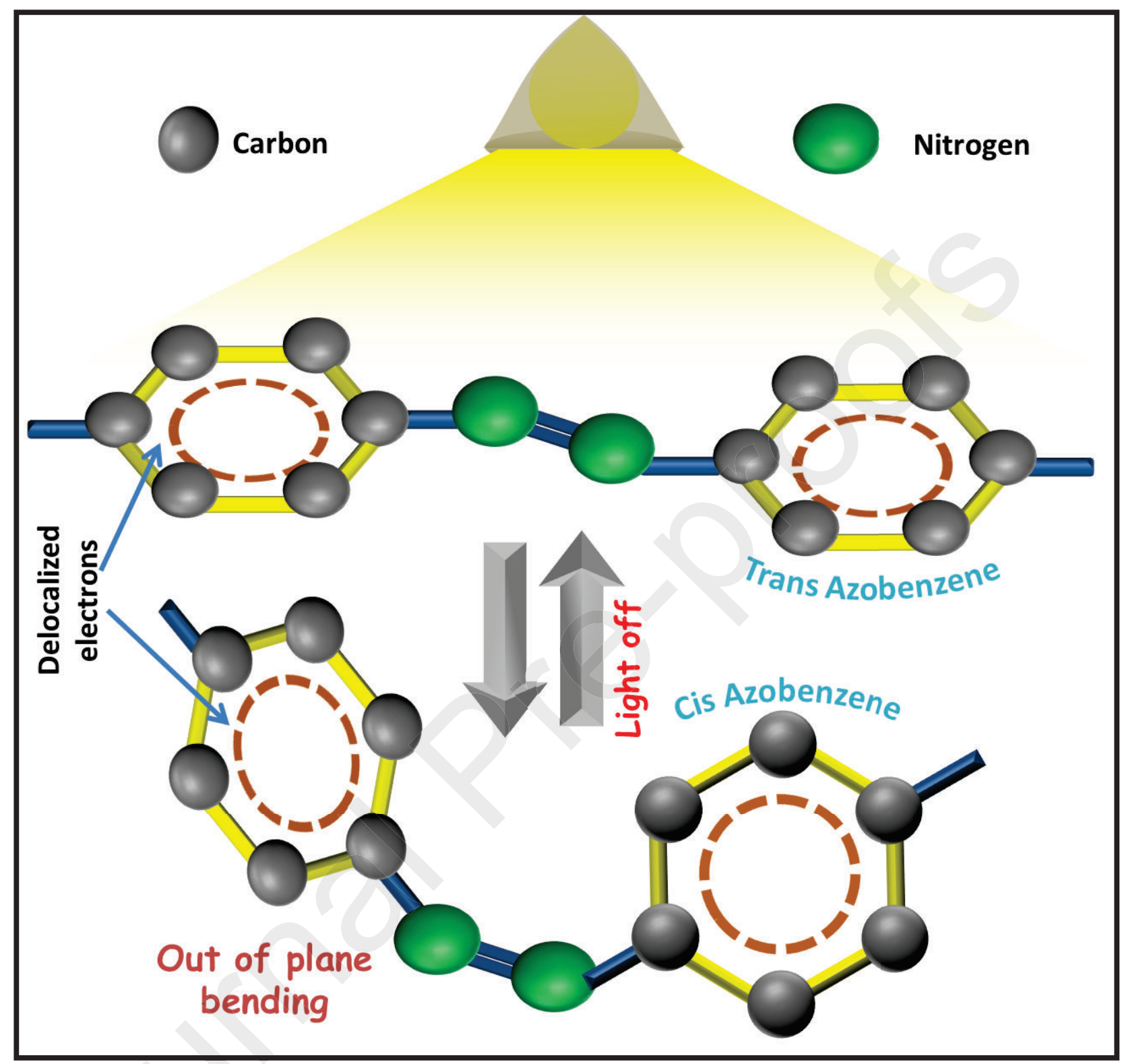

Figure 8. Systematic representation of azobenzene actuation in light stimulus.

Dyes containing oxygen $\left(\mathrm{O}_{2}\right)$, nitrogen $\left(\mathrm{N}_{2}\right)$ and sulphur $(\mathrm{S})$ atoms have small band-gap which aids in repositioning of absorptions bands in IR region. In croconaine dyes, absorption in nearIR region (with aforesaid mechanism due to transitions in band) is also encouraged by delocalization of negative charge from $\mathrm{O}$ along the backbone (equipping the dye with conductivity). Absorption by these dyes can adapt different responses which is relied on substitution e.g. metal (zinc ( $\mathrm{Zn}$ ) can shift $871 \mathrm{~nm}$ to $788 \mathrm{~nm}$ ) and thiophene. Photon 
bombardment on molecules increases heat energy kinetically which is conducted along the chain through delocalization. This phenomenon combined with Beer Lambert's law, conductivity and hyperthermia escalateT in its (material)vicinity to higher degrees which is sufficient to drive photothermal activation for pre-agreed applications (Figure 9) [58-60]. Spence et al. [60] delineated the accessibility of Beer Lambert's law with $\Delta \mathrm{T}$ ( $\mathrm{T}$ rise) for croconiane dye and explained that for predetermined incident wavelength $(780 \mathrm{~nm})$, corresponding power $(250 \mathrm{~mW})$ (density- $\left.3.5 \mathrm{~W} / \mathrm{cm}^{2}\right)$ and conc. $(2 \mu \mathrm{g} / \mathrm{mL}), \Delta \mathrm{T}$ initially increases which finally forms a plateau for maximum absorbance.

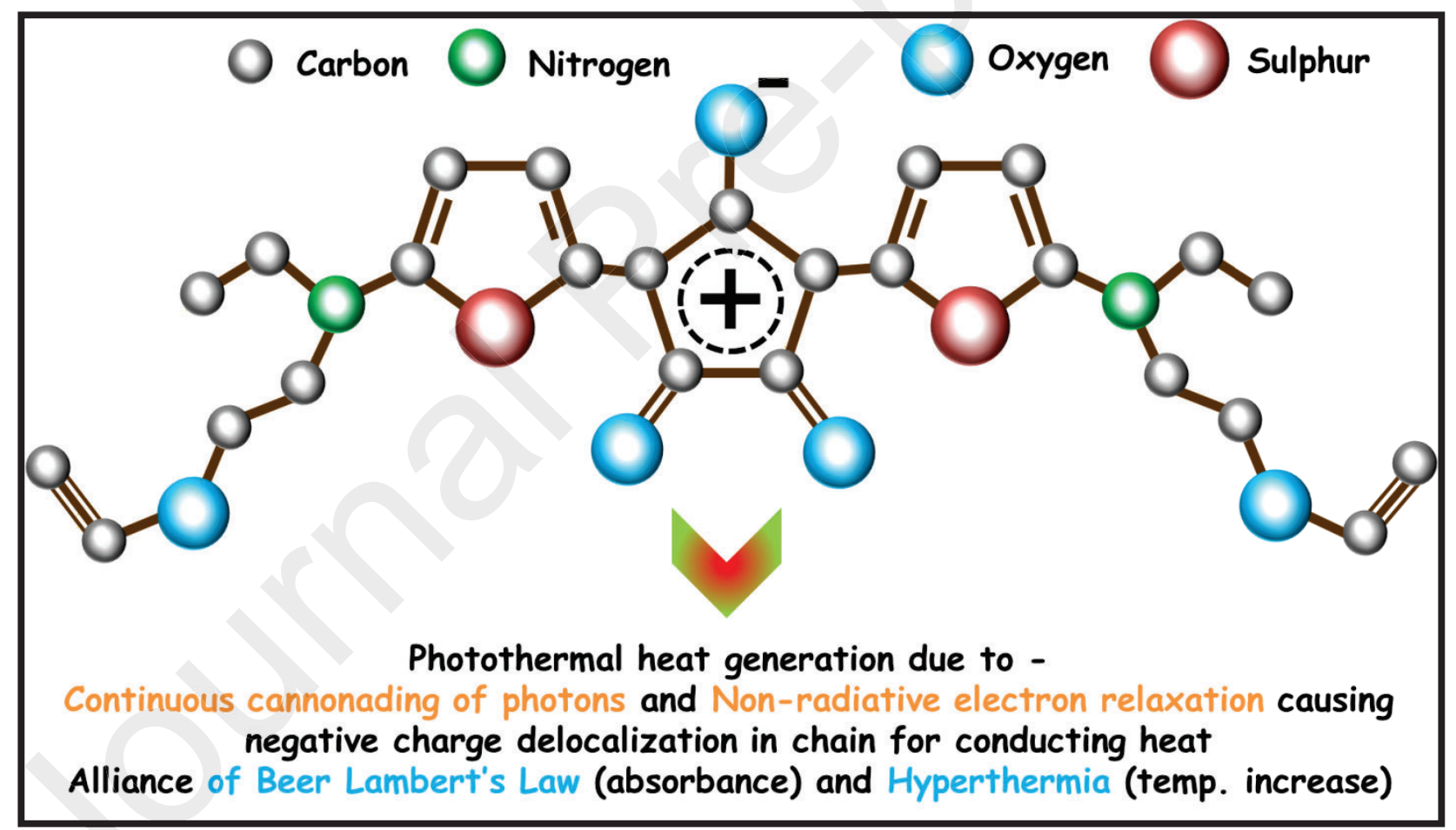

Figure 9.Systematic representation of croconaine dye and its photothermal mechanism.

However, in metal-nanoparticels (NP) e.g. Au, Ag (research interest for medical therapies) (size range $=10-200 \mathrm{~nm}$ ), photothermal property inheritance is attributed to Localized Surface Plasmon Resonance (LSPR) (Figure 10). Metals are accompanied by sea of electrons; therefore, light interaction aids in breaking of its loose attachment with orbitals thus freeing 
them to occupy higher unoccupied levels (known as hot electrons). When the wavelength associated with these conducting electrons forms resonance with incident IR, amplitude of vibration increases at same frequency and communicates with electric field vector of IR. Resultant dipoles oscillate with moving electric field and bestow heat which is generated due to mass collision of electrons with electrons/lattice/phonons $($ time period $=$ fraction of a second) along with contribution from inelastic interaction of light with NPs [40,61-67]. This occurs because dipole oscillation is highly localized phenomenon, therefore, enclosed energy field interacts with adjacent alike from another atom and process follows resulting in amplification of electric field energy which is higher than that associated with incident energy. This is known as LSPR or alternatively it can be defined as when incident intensity gets captured at nanoscale through light-matter interface which can readily be channelized for appropriate fulfillment of operations. LSPR in metals was being acknowledged since 400 A.D. especially in noble $\mathrm{Au}$ and $\mathrm{Ag}$ nanoparticles whose free electron concentration $\left(\sim 10^{22} \mathrm{~cm}^{-3}\right)$ has customized resonant frequency in human-perceivable range engendering varied optical effects. Larger or intricate geometries tends to red- or blue- shift resonance beyond visible range e.g. resonance with aluminium was observed near UV-Vis range but still governing factors (size, shape etc.) limit exists (size < wavelength of incident light) beyond which no LSPR can be witnessed. Nonetheless, density of charge carriers also circumscribes their optical range but with progression to LSPR in semiconductor nanoparticles, scientists can widen the range by piloting dopants $\left(10^{19}-10^{21} \mathrm{~cm}^{-3}\right)$. LSPR of metals have resonance quality factor approximately $10-20$ and is defined as ratio of resonance wavelength to resonance width (Equation 1). 
Resonance quality factor $=\frac{\lambda \text { resonance }}{\Delta \lambda}, \Delta \lambda=$ resonance width

This quality factor can be ameliorated or $\Delta \lambda$ can be narrowed with apt combination of shape and size (as aforementioned) along with its periodic arrangement in a phase relationship. These are widely been explored for its employment in medicines, nanoelectronics, optics, nanotweezers, photovoltaics, imaging in addition to other engineering fields [66,67].

Localized hyperthermia is transferred in its neighborhood which assist in the uplifting of macroscale $\mathrm{T}$ to tens' of degrees $\left(36-60^{\circ} \mathrm{C}\right.$ for $8-32 \mathrm{~W} / \mathrm{cm}^{3}$ laser) subjecting the particle to selectively eradicate vicious cancer cells owing to its $\mathrm{T}$ sensitivity. Hyperthermia or photothermal conversion efficiency is a function of absorbed wavelength which further displays sensitivity among dimension, shape and concentration. Dimensions along with nature of incident light determine occurrence of LSPR in NPs e.g. Au nanorods with aspect ratio $=$ $2.4 \rightarrow 6.6$ can absorb wavelength $=640 \rightarrow 1050 \mathrm{~nm}$ respectively, similarly, core-shell ratio or shell thickness, and diameter of Au nanospheres also assist in modulation of incoming light energy for photothermal response. In terms of shape, nanorods display 2-3 fold increased absorption when compared to nanoshells \& nanospheres and conc. proportionate with $\mathrm{T}$ elevation. Au for medical treatment is accredited to its inertness, biocompatibility, nontoxicity, nano-heaters resistant to photobleaching etc. Conclusively, absorption of light for a material depends on innate electrons, their orbital structure and transition gap, accordingly, photothermal efficiency of $\mathrm{Ag}>\mathrm{Au}$ which is bestowed by large energy band gap in $\mathrm{Ag}$. Additionally, optimization in structural profile of Au-NP (i.e. shell, sphere, rods etc.) can showcase photon $\rightarrow$ heat efficiency conversion from $22-103 \%$ [40,61-67]. 


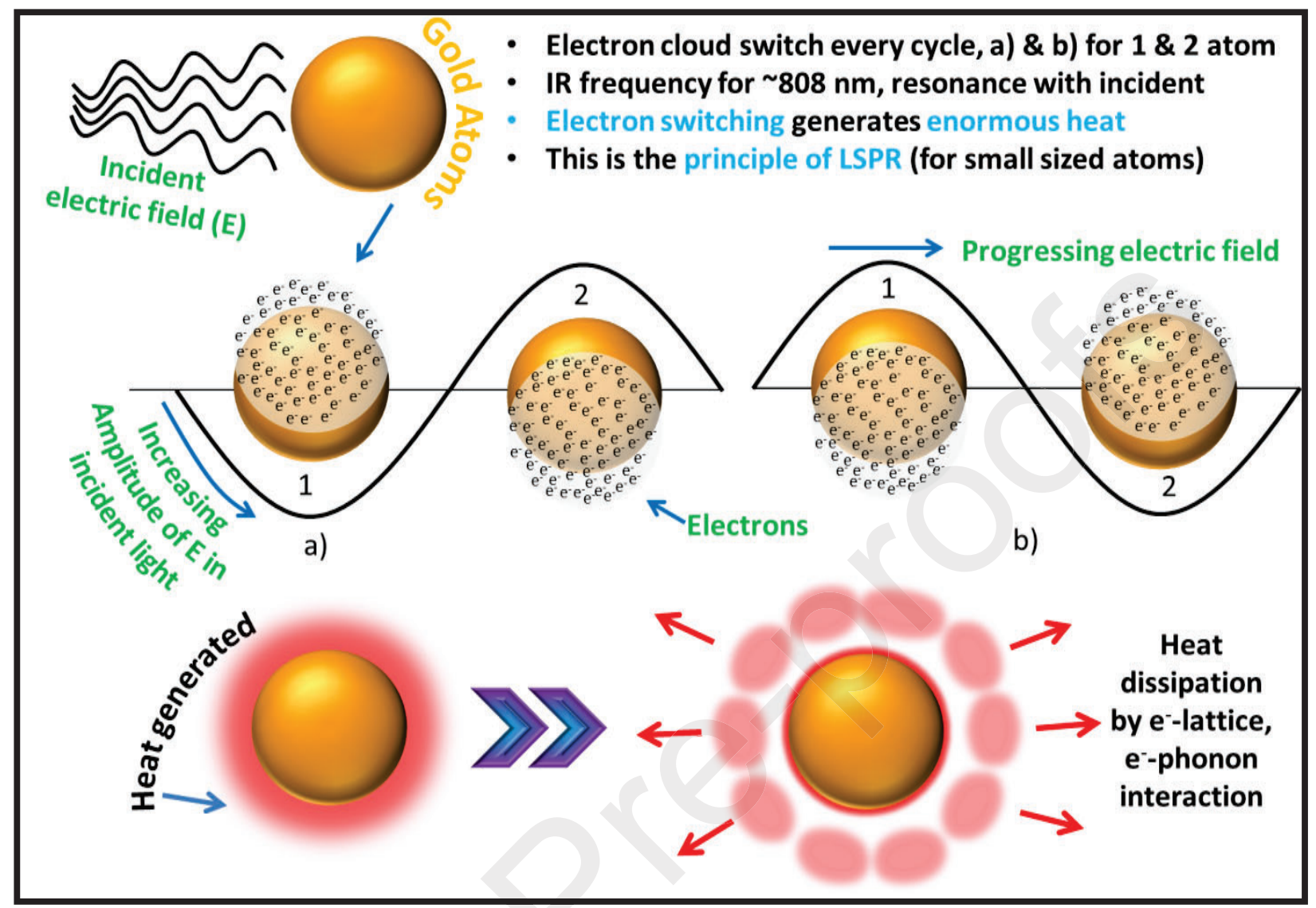

Figure 10. LSPR mechanism in metal $(\mathrm{Au})$ nanoparticles.

Akin to above mechanisms, photon bombardment on graphene energizes orbital electrons initiating innumerous electron-hole pairs which are carriers of heat energy i.e. hot electrons (photon energy $\rightarrow$ hot electrons (in which energy $>$ Fermi level energy)). These hot electrons through various interactions with electrons, phonons, and lattice, relax back to their low energy stable state with energy emission due to cooling effect (irrespective to azobenzene which favors conformation change instead of emission). This emitted energy to surrounding foster hyperthermia (medical attention e.g. cancer) which is accelerated by pre-eminent thermal conductivity of electrons thus causing10's of degrees increases in surface temperature. Further, this cycle continues and photons multiplicably generate more conducting pairs with manifold local burgeon in temperature and thereby, in its surrounding. 
Like graphene, CNTs follow the footstep mechanism of graphene in photothermal generation of heat through electron-photon scattering and delocalization of $\pi$-electrons. Absorbed energy, as in above literatures, can be attuned by various doping e.g. O having an ability to shift wavelength to IR and thus accessible for medication [68-71]. Therefore, electronic interactions (absorption and conversion associated with bandgaps, atomic weights etc.) of material with incident energy (stimuli) acts as fingerprints to characterize the samples.

\section{Photothermal LCs}

Former section elucidates about the molecular reaction of LCs in relation to external stimulation of light which is proficient in transforming the incident energy to thermal actuation for perceptible macroscopic change. To activate the LC for transformation, multitude of alternatives can be accessed, for instance, T, magnetic field, light, electric actuation etc. but light (either Visible (Vis), Ultraviolet (UV), or infrared (IR)) can cater stimulation for far-flung areas through radiation assistance [72]. The significance of photothermal stimulation can be comprehended by its propensity to trigger under extreme non-contact environments. In addition to the former, it also empowers the operator to control preciseness of impinging radiations location (thereby causing heating) and its illuminating time (efficient in being able to on/off on desire) for LC's remote functioning $[73,74]$. For the aforementioned reasons, various reinforcements e.g. metal-NP (gold (Au) and Ag), inorganic and organic molecules (carbon nanotubes, dyes, graphene, polydopamine, etc.) are indispensable which functions as nanoscale heat source [75].

\subsection{Carbon-based materials in LCs}


Section 2 has unfolded the molecular interactions of various carbon-based materials (graphene and carbon nanotubes (CNTs)) which equip them with efficacy in heat production from incoming photons and its subsequent conduction along the structural path. Rapid generation of heat with the illumination of UV/near-IR/Vis and its swift dissipation in surrounding arena on switching off of an illuminating source has engendered carbon-based materials for multitude of applications in actuators and medicals [41]. LC elastomers with graphene dispersion (reduced from graphene oxide (GO) to varied proportions $\sim 55 \%$ and $\sim 66 \%$ ) can skillfully respond to near-IR (808 nm), visible light, or natural energy from sun to produce extension/contraction in merely seconds with $\mathrm{T}$ changing by factor of around $80{ }^{\circ} \mathrm{C}$ or oscillating around the value in a range [76,77]. Quantitatively, reduced surface groups in GO impacted not only transformation stress but also produced strain (which is proportional to $\mathrm{sp}^{2}$ carbon (C) states) moreover, $\mathrm{sp}^{2}$ hybridization also assisted in fastshipping of heat to surrounding assisting in conduction [77]. In addition to hybridized C state, intensity and magnitude of incident stimulus also manipulate the transformational output (i.e. T, response time, mechanical properties etc.). Wang's group [78] had functionalized graphene with mesogens (C6M, chiral smectic $\rightarrow$ cholesteric) and observed that resultant could be actuated with different stimuli namely, light (sunlight $\left(35^{\circ} \mathrm{C} / 22^{\circ} \mathrm{C}\right)$, near-IR $(980 \mathrm{~nm})$, and Vis $(700$ $\mathrm{nm})$ ) and voltage $(18 \mathrm{~V})$. Furthermore, stimulation time was altered by incident trigger i.e. power/voltage $\propto$ response/recovery time for transparency/opaque property and magnitudinally for $0.5 / 3 \mathrm{~W}$ near-IR $\rightarrow$ time required $\sim 2 \mathrm{~min} / \sim 60 \mathrm{sec}$ respectively. An improvement of several folds was obtained with GO/azobenzene in LC which permitted actuation in UV/Vis/nearIR/sunlight. The response time-period had shortened to $1 \mathrm{sec}$ in UV \& near-IR while $1.5 \mathrm{sec}$ for Vis with $1 \mathrm{sec}$ relaxation time. Furthermore, with experimentation, it was deduced that 
change in azobenzene concentration could vary the isotropic transition time hence, $5 \mathrm{wt} \%$ (having fastest transition among experimented concentration) was chosen for the composite formulation. This GO + azobenzene drove bending under different light illumination (UV/near-IR/Vis) (maximum bending at 120\% elongation ratio due to orderly alignment of all mesogens and further increment in elongation ratio had not shown any improvement in bending) [41].

Second carbon-rooted and substantially employed nanomaterial is CNTs which can be visualized as directionalized rolling of graphene's flat sheet which alike its counterpart (graphene), is proficient in switching heat from light and its shipping to far-off distance with a condition that it is homogeneously dispersed in LC (practical shortcoming for researchers) [79]. Agglomeration poblem is attributed to intra-CNT Van der Waal's forces which shadow other interactions and can be overcome by its functionalization which induces stabilizing effect on its surface and aid in the attainment of consistent conc. in a volume. However, pristine single-walled CNTs (SWCNT) witness magnification in resultant properties over its multi-walled counterpart [80]. For instance, Yang and companions had functionalized SWCNT with aromatic polymer (poly (p-phenyleneethylene) with $<0.2 \mathrm{wt} \%$ dispersion which otherwise (higher conc.), would result in hinderance for reversible movement of side chains mesogens during nematic $\leftrightarrow$ isotropic transformationsresulting in uncontrolled heat increment [81]. This conc. restriction was overcomed by Ji et al. with $\leq 3 \mathrm{wt} \% \mathrm{CNT}$ in pyrene/LC with main chain configuration thus, enhancing dispersion (without intervening chain movements) but $3 \mathrm{wt} \%$ resulted in phase separation which was indicated by $3^{\text {rd }}$ transition point (in addition to solid-liquid and nematic-isotropic peaks) and a depletion in achievable strain [82]. Aside from its thermal properties and non-contact actuability, CNT reinforcements are also a 
powerful source to mechanically stabilize (toughen, modulus, strength, stiffness, elasticity, etc.) the composite accomplishing multi-functions [20]. The improvement was witnessed in modulus and fracture strength in tension for $<0.8 \mathrm{wt} \%$ reinforced SWCNT beyond which the sharp declination was evidenced [46,81]. Moreover, the stress necessitated for transition and consequent deformation was ascertained as a function of applied illuminating intensity and wavelength showed augmentation for predefined CNT concentration [46,83,84]. Marshall et al. noticed dwindling in stress value when incident wavelength was rolled towards near-IR from visible i.e. stress magnitude followed $2 \rightarrow 2.3 \rightarrow 1.9 \mathrm{kPa}$ when illumination ranges by $670 \rightarrow 785 \rightarrow 980 \mathrm{~nm}$ respectively [83]. Li et al. lit white light on LC-elastomer in range $3.4 \rightarrow 413 \mathrm{~W} / \mathrm{cm}^{2}$ power density which resulted in shifting of stress value, $80 \rightarrow 75 \mathrm{kPa}$, due to attainment of saturation of aligned mesogens. Further, they added that composite temperature had risen to $90^{\circ} \mathrm{C}$ while $36^{\circ} \mathrm{C}$ was observed for solely LC-elastomer only in a period of $10 \mathrm{sec}$ [46].

However, the not so widely explored photothermal function is the welding/healing domain has been achieved in LC-Vitrimers. Vitrimers are class of polymers which epitomize a compromise between thermoplastic reusability or reprocessability (at $>$ transition temperature, which supports viscoelasticity) and thermoset strength with covalent crosslinkability (at < transition temperature) in addition to its improvement in high-temperature stability. Liquid crystal vitrimers undergo reversible bond-exchange reaction of transesterification (renewable of ester and $-\mathrm{OH}$ bonds from their old bonds) for renewing the broken bonds bestowing reprocessability to prepared polymers $[85,86]$. Additives diversify actuation region as instance, Yang et al. propounded CNT as a micro/nano-scale heat source (photothermal actuation) working in unison with LC-Elastomer for photon aided welding, design actuation, and healing 
(Figure 11). They depicted various design e.g. film, chair, weight-carrying person etc. having an actuation time within $20 \mathrm{sec}$. When further experimented for healing, time, it acquired, was dependent on size of crack i.e. for crack dimension in order of $93 \mu \mathrm{m}, 126 \mu \mathrm{m}$, and $220 \mu \mathrm{m}$ (hole) response appeared in 5, 10, and $10 \mathrm{sec}$ respectively without compromising the mechanical strength. Re-attainment of mechanical feature was observed in addition, when the detached part of a composite entity was photo-welded without disturbing the alignment of mesogens in an unexposed region meanwhile, curtailing the time of welding to seconds. Authors had also deduced compelling CNT strength, being able to elevate temperature from $130^{\circ} \mathrm{C}$ to $180^{\circ} \mathrm{C}$ while avoiding fatigue even after 20 cycles, and could raise weight in 1000 multiplication factor [87]. Howbeit, CNT aggregation extensively impacts localized mesogen alignment, hyperthermia, as well its interfacial interaction with LC matrix which are primly desirable for final performance of material and device. The challenge was subjugated by employing polydopamine as an additive in LC-elastomer undergoing transesterification bondexchange reaction (or LC-vitrimer). Polydopamine displayed similar characteristics i.e. photothermal actuation, and photo- welding, healing with ease in dispersion attributed to hydroxyl $(-\mathrm{OH})$ and amino group favoring hydrogen bonding (covalent crosslinking). Therefore, $2 \mathrm{wt} \%$ polydopamine dispersed in size of $<20 \mu \mathrm{m}$ demonstrated transition temperature of $105^{\circ} \mathrm{C}, 350 \%$ strain at failure than $25 \%$ in neat LC. Additionally, it evidenced proportional increase in temperature with NIR power i.e. $110^{\circ} \mathrm{C}-0.85 \mathrm{~W} / \mathrm{m}^{2}$ and $160^{\circ} \mathrm{C}-1.4$ $\mathrm{W} / \mathrm{m}^{2}$ in $15 \mathrm{sec}$ irradiation. Light invigorated NIR-induced healing in $15 \mathrm{sec}$ for hole diameter of $600 \mu \mathrm{m}$ or scratch or $100 \mu \mathrm{m}$ wile welding initiated after $8 \mathrm{~min}$ at $200^{\circ} \mathrm{C}$ in conjunction with pressure [86]. Conveying the similar notion, LC-Vitrimers can also employ aniline trimer based photothermal additive for exchange bond reactions further easing agglomeration 
concern [85]. Pei et al. demonstrated micropattern (at $240^{\circ} \mathrm{C}$ ) recovery for one-way SME in strip containing epoxy (without LC structure) and LC-Vitrimer (decanedioic acid and epoxy having biphenyl mesogens) layers at temperature $>\sim 160^{\circ} \mathrm{C}$. While triple SME was achieved at temperature of $130^{\circ} \mathrm{C}$ deformed and cooled to first temporary shape at $75^{\circ} \mathrm{C}$, further to $<55^{\circ} \mathrm{C}$ for second temporary shape and memory regained in heating cycle traversing across same temperature points [88].

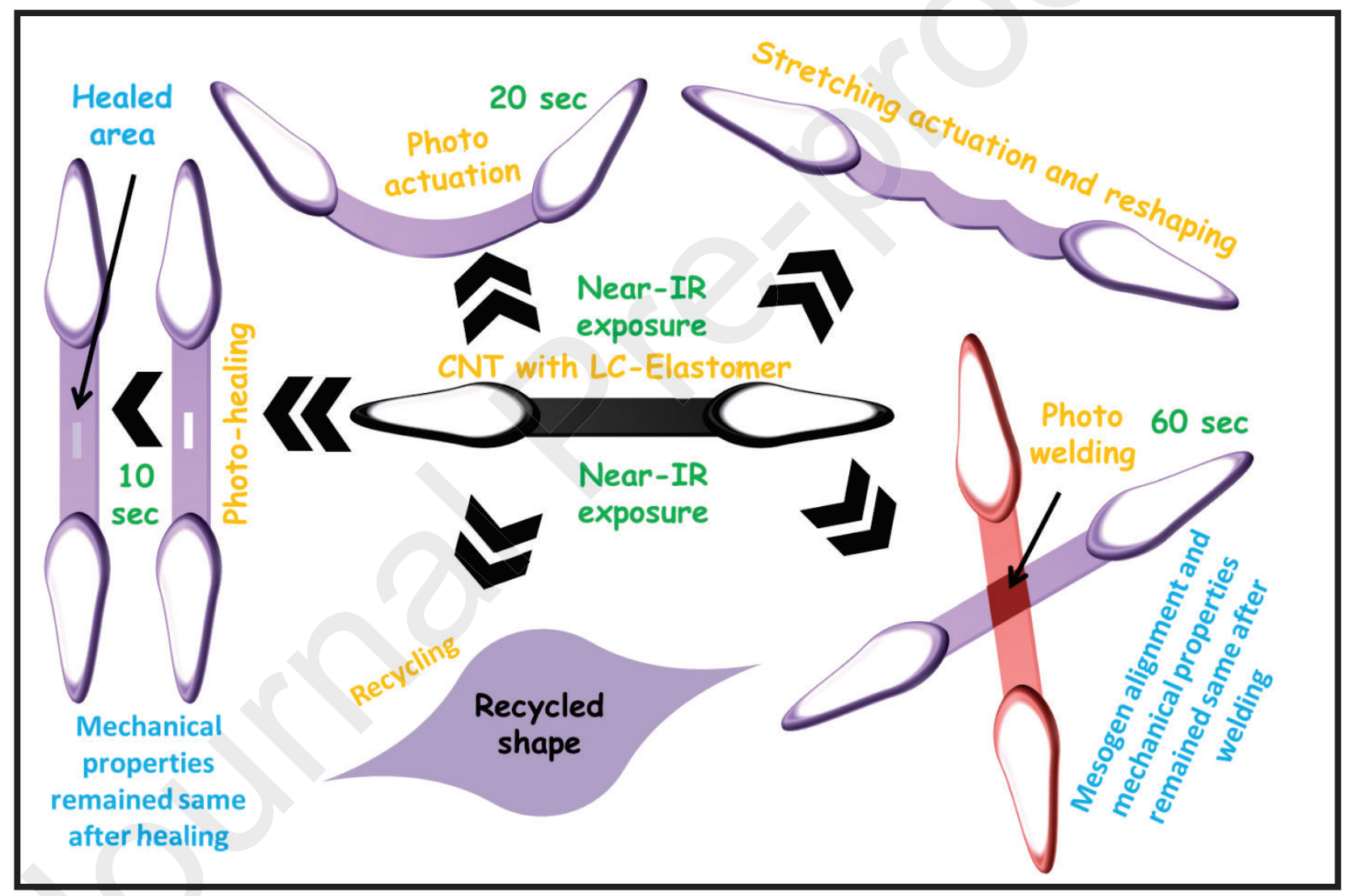

Figure 11.Different functions exhibited by CNT dispersed LC-elastomer.

Azobenzene experiences conformational variation in light but with thermal and UV aiding (i.e. external $\mathrm{T}+\mathrm{UV}$ generated heat), it can also transform photothermally which functions to expand its performance range $[41,82]$. Further analysis also volunteer shape memory in the 
formulated composite which shuttles between room-T to $110^{\circ} \mathrm{C}$ and $\mathrm{UV}$-aided transformation with azobenzene evidenced slower trans recovery force from cis state due to photothermal effect [89].

\subsection{Dyes activated LCs}

Dyes-functionalized LC bestowed organic-organic compatibility in formulated solution which is superior to inorganic-organic interaction in metal-NP and also confers effective photontemperature conversion without deteriorating mechanical and dispersion uniformity of composite $[54,90]$. Dyes whose potency is discussed in literature are- croconaine, lumogen IR788 [54], dispersed red-9 [91], phenol blue [92], pyrromethene-650 [93], polyaniline[94] etc. Influence of LC nematic-isotropic configuration on responsive behavior due to photothermal is also exploited in dielectric study of holography where refractive and diffraction efficacy with dye concentration was considered [91]. Refractive index reliance on photothermal effect in dielectric research was attributed to the molecular disturbance that took placewhen the structure moved towards randomness which diffracted incident light [92]. In regards with absorption phenomenon, croconaine dye allied with acrylate-based LC-elastomer was negatively strained (shrinkage) to $25 \%$ in $\sim 33 \mathrm{sec}$ transcending $\mathrm{T}_{\text {trans }}\left(\sim 106{ }^{\circ} \mathrm{C}\right)$ and regained back its original form in $<5 \mathrm{sec}$ owing to reversible crosslinking driving force [54].

Another material that has captured the limelight of photothermal actuation domain is aniline, though an aromatic compound but has capability to energize its surrounding from the incident photons. Exploiting the conjugation and subsequent electronic transitions, aromatic structure of aniline absorbs light in near-IR region and engenders photothermal effect [94,95]. However, extent of effectiveness can be inferred by its conversion rate (photon $\rightarrow$ heat) which 
was observed low and therefore, conjugation structures were examined e.g. emeraldine salt/base because protonated structure functions to shift its absorption range. Unlike weight sustenance ( 200 times), concentration and conjugation features alter conversion efficacy i.e. salt analog shrank faster (20 sec) over its base counterpart $(26 \mathrm{sec})$ and also actuation rate was proportionally accelerated with conc. (as 1, $0.5 \mathrm{wt} \% \rightarrow 16,20 \mathrm{sec}$ respectively) [94]. Analogous to aforesaid photo welding/healing in CNT, aniline LCs had also undergone similar study and outcome was comparable with the previous as both reinforcements required $10 \mathrm{sec}$ for healing but aniline utilized lower intensity than CNT (as $0.66 / 0.84 \mathrm{~W} / \mathrm{cm}^{2}$ for aniline/CNT respectively) and also $\leq 30 \mathrm{sec}$ for welding under light irradiation. Additionally, study also corroborated multi-shape memory at different intensities optimized at varied temperature. Driving stress for shape change vary proportionally with incident light strength of same wavelength (acts as motivation for multiple shapes) [85]. This principle can be availed in biomedical and actuators engineering by employing different wavelengths and powers. Zeng et al. had demonstrated LC-elastomer with inverse actuation (i.e. more light, more contraction in iris and vice-versa) by steering strain during photopolymerization which was due to aniline photon-sensitivity (when $\frac{T_{\text {polymerization }}}{T_{\text {room }}}>1$, relaxation of chains (or thermal expansion) induced bending in the printed material and when this governing ratio was greater, larger \& faster bending steered the structure until complete molecular orientation, thus driving reverse actuation in light). However, photothermal realization originated with dye named dispersed Red-1 which circumvented UV and shifted actuation in visible wavelength (488 $\mathrm{nm})$. The researchers designed iris having modulus of 2.5 MPa which effectively barricaded transmission with increasing light power $(30 / 180 \mathrm{~mW}$ allowed $90 \% / 30 \%$ light respectively) 
and its swiftness was guided by power density ( $<5 \mathrm{sec}$ with $300 \mathrm{~mW} / \mathrm{cm}^{2}$ and $30 \mathrm{sec}$ with 230 $\mathrm{mW} / \mathrm{cm}^{2}$ ), the study was proposed for smart recognition in optics [22].

\subsection{Metal Nanoparticle activated LC}

NPs of metals e.g. copper, palladium, Au, platinum, and Ag have spellbound characteristics like acting as one-millionth scale heat sources (surface plasmon resonance (SPR)) and nonlinearity in optics of $3^{\text {rd }}$ order which has made scientists fascinated about their engineering and medical (e.g. tumor medication etc.) exploitations [40,44]. Nanoscale, where properties rollercoaster for even minute parametric changes, necessity arises for modeling e.g. when spheres of $\mathrm{Au} \& \mathrm{Ag}$ mimic the dimension of molecules i.e. 1-2 $\mathrm{nm}$, their characteristics resemble and SPR transpire [96]. Pezzi et al. had modeled Au NPs which demonstrated transitions depending on internal energy and temperature in relation with oscillating electrons that was due to incident wavelength followed by their aftereffects on refractive index (RI) and polarizability. In addition, authors noticed that experimentation results corresponded to model prediction with $\mathrm{RI}_{\text {isotropic }}=1.56[97]$. Surface of Au NPs could be tailored with thiol for stabilization and additionally rendered crosslinking with siloxane-backboned LC-elastomer ushered increased stiffness to composite (Figure 12) [98]. Further, Au NPs can take myriads of forms e.g. rods, spheres, shells, etc. among which rods and spheres had been researched by Liu et al. [99]. Authors unraveled that for $1 \mathrm{wt} \%$ of either(rods \& spheres) in composite pillars, temperature rise differentiated efficiency in heat production i.e. $\Delta \mathrm{T}_{\text {rods }}=48^{\circ} \mathrm{C} \& \Delta \mathrm{T}_{\text {sphere }}$ $=12^{\circ} \mathrm{C}(\Delta \mathrm{T}=\mathrm{T}$ rise $)$ and thus $\mathrm{Au}$ rods were inferred to surpass spheres in LC-Elastomer for targeted heating [99]. Understanding of shape memory effect, conc.and dimension are also fundamental variables forculminating properties. First, size effect, which was found as 
decisive factor for absorption range i.e. it can tune incoming radiation to Vis/IR region. With spherical particles absorption plot manifested single peak however, rods exhibited two peaks corresponding to its long and short axis respectively thus, the signal could be modulated in wake of its aspect ratio. Experimentations revealed that size enlargement had favored absorption shift towards higher wavelength (Table 2) [30,97,99,100].

Table 2. Absorbed wavelength as a function of particle dimension and morphology.

\begin{tabular}{|c|c|c|c|c|c|}
\hline Particles nature & \multicolumn{2}{|c|}{$\operatorname{Dimension}(\mathbf{s})(\mathbf{n m})$} & \multicolumn{2}{|c|}{$\begin{array}{l}\text { Absorbed wavelength(s) } \\
\qquad(\mathrm{nm})\end{array}$} & References \\
\hline \multirow{2}{*}{ Sphere } & \multicolumn{2}{|c|}{15} & \multicolumn{2}{|c|}{520} & [99] \\
\hline & \multicolumn{2}{|c|}{$17.4 \pm 2.2$} & \multicolumn{2}{|c|}{526} & [97] \\
\hline Rods & $50-60$ & $8-10$ & 865 & 510 & {$[30]$} \\
\hline M6S/Au rods & $44.26 \pm 3.74$ & $14.75 \pm 1.75$ & Around 720 & Around 530 & {$[100]$} \\
\hline
\end{tabular}

$\mathrm{M} 6 \mathrm{~S}=\mathrm{Au}$-nanorods with hydrophobic single-layer of mesogenicthiol

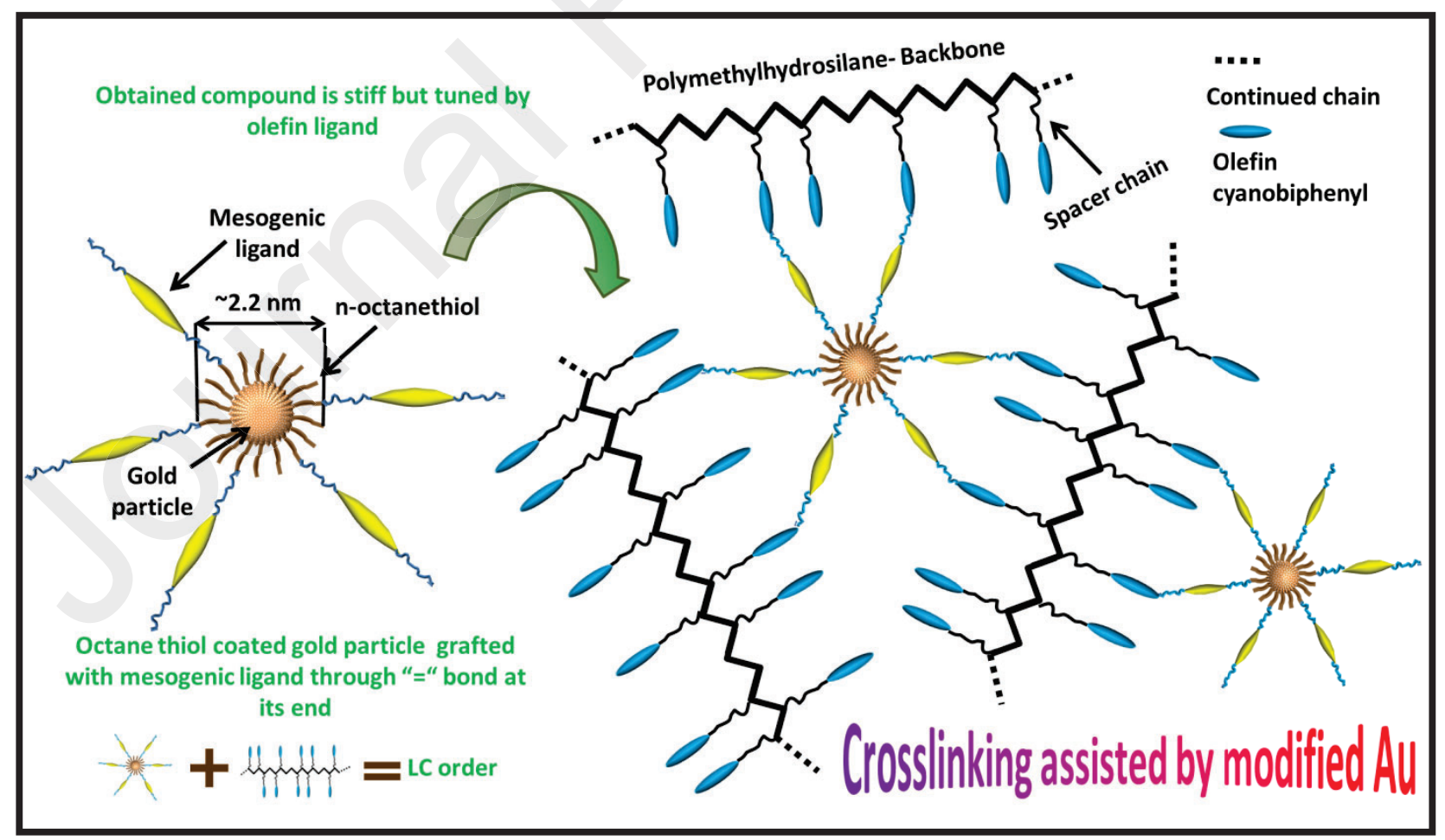

Figure 12. Picturization of modified Au which acts as crossliner to form LC. 
Smaller magnitudes of 720 and $530 \mathrm{~nm}$ wavelength (strong long axis and weak short axis absorption) for corresponding 44 and $15 \mathrm{~nm}$ dimension with LC M6S/Au as opposed to 865 and $510 \mathrm{~nm}$ for $50-60$ and 8-10 nm was expected due to Au rod surface modification which has also been observed by Liu et al. and Yang et al. [30,99,100]. Second, concentration effect, an apt source governing $\Delta \mathrm{T}$ and its greater value facilitates room- $\mathrm{T}$ irradiation for (smectic)nematic-isotropic transition within seconds or a fraction. Yang et al. had incorporated 0.09 $\mathrm{wt} \%$ of Au nanorods and reported that perceptible change was delayed to $\sim 10 \min [30]$ and similar results (governingrelation) were obtained with $0.01 \mathrm{wt} \%$ [100]. Gutierrezcuevas et al. discovered that $0.2 \mathrm{wt} \% \mathrm{LC}$ responded in $7 \mathrm{sec}$ when $\Delta \mathrm{T}=4^{\circ} \mathrm{C}$, however, when difference was further widened $\left(15^{\circ} \mathrm{C}\right)$, entire transition barely occurred [100]. Moreover, conc. increase agglomeration in Au NPs and accumulated heat after irradiation was sufficiently discernible as $1 \mathrm{wt} \%$ had randomized $\mathrm{LC}$ to isotropic state in $5 \sec \left(\Delta \mathrm{T}=115-30=85^{\circ} \mathrm{C}\right)$ with recuperation in $1.5 \mathrm{sec}$ [99]. Pezzi et al. experimented with increased concentration of up to $6 \mathrm{wt} \%$ ascertained that $3 \mathrm{wt} \%$ was the peak surpassing which LC's ordering is challenged [97] but Kuang et al. presented unequal distribution even beyond $1 \mathrm{wt} \%$ despite that LC composite displayed improvement in hydrophobicity with concentration [101]. This discrepancy could be accounted to functionalization difference as Pezzi et al. capped Au particle with surfactant. 0.4 $\mathrm{wt} \% \mathrm{Au}$ LC samples had also been appraised for its photo-healing potential being able to wipe away crack in $5 \mathrm{sec}$ [102].

Alternative to $\mathrm{Au}$ is $\mathrm{Ag}$ where absorption efficiency for SPR is superior due to additional resistance to electrons in the flow pathway [40]. Resonance absorption was spotted on $460 \mathrm{~nm}$ for $12 \mathrm{~nm}$ particle dimension, but LC/Ag processing was challenged when cyanide presence at 
side chain reacted with $\mathrm{Ag}$ forming $\mathrm{Ag}-\mathrm{CN}$ and destroying LC effect. State was averted by amalgamating thiol group in polymer chain which initiated its attachment on Ag surface and stabilizes LC/Ag [103]. Following the Ag-S bond for surface compatibilization in LCelastomer, $\mathrm{T}_{\text {trans }}=\sim 55^{\circ} \mathrm{C}$ and stimulated strain was $\sim 25 \%$ in $15 \mathrm{sec}$ but extended to $30 \%$ in 80 sec while recovering back in $80 \mathrm{sec}$. Furthermore, Ag concentration augmented $\Delta \mathrm{T}$ and strain i.e. for 0.1 and $0.2 \mathrm{wt} \%$ detected strain was $\sim 20 \%$ with $\Delta \mathrm{T}=52^{\circ} \mathrm{C}$ while $\Delta \mathrm{T}=48^{\circ} \mathrm{C}$ with $10 \%$ deformation having $0.04 \mathrm{wt} \%$ concentration was witnessed. Moreover, $\Delta \mathrm{T}<\mathrm{T}_{\text {trans }}$ which could be amplified by elevating the incident wavelength intensity to $1.5 \times 10^{5}$ lux. The study also suggested that tensile strength for $0.1 \mathrm{wt} \%<0.2 \mathrm{wt} \%$ by $40 \%$ [104]. Comparing this study with Pezzi et al. Au LC revealed that although with similar $\mathrm{T}_{\text {trans, }}$, actuation time was half and relaxation was one-fourth to that in $\mathrm{Ag}$ which may be attributed to low band gap and high conductivity in $\mathrm{Au}[97,104]$.

Other metal that can be photothermally actuated is Nickel (Ni) which after forming complex with thiolene group occupy $\sim 960 \mathrm{~nm}$ peak when irradiated (owing to large band gap compared to $\mathrm{Au}$ and $\mathrm{Ag}$ ). Illumination triggered $\Delta \mathrm{T}$ to $215^{\circ} \mathrm{C}$ within $70 \mathrm{sec}$ at $2.5 \mathrm{~W} / \mathrm{cm}^{2}$ and time $\mathrm{e}_{\text {actuation }}$ could be modulated with power density (at actuation $\mathrm{T}$ of $110^{\circ} \mathrm{C}$, response time was $37 \mathrm{sec}$ at power $1 \mathrm{~W} / \mathrm{cm}^{2}$ while actuation duration reduced to $3.5 \mathrm{sec}$ at same $\mathrm{T}$ when power was increased to $2.5 \mathrm{~W} / \mathrm{cm}^{2}$ ) with proposed applications in data storage, sensors etc. [105].

In summary, studies on photothermal LC with carbon-related/metal NP/dyes are recapitulated as follows:

- $\mathrm{T}_{\text {trans }} \propto$ (reinforcement) $)^{-1}$, fornematic-to-isotropic or trans-cis attains a lower numeric which eases overall process. 
- Azobenzene along with CNT/Graphene/Dye/Metal-NP gave an additional feature to actuate in wavelengths spanning over UV/Vis/Near-IR.

- $\quad$ Power density $\propto\left(\text { time }_{\text {transition }}\right)^{-1}$.

- $\mathrm{LC}+\mathrm{CNT} / \mathrm{Graphene} / \mathrm{Dye} /$ Metal NP are quintessential for transformation, solely LC cannot retaliate to incoming trigger.

- Homogeneous dispersion and distribution (usually by ultrasonication) of photothermal source in minute quantity (conc.) are imperative for entropic change leading to macroscopic recognizable variation and effective photon conversion.

- Illuminating surface of composite design volumetrically changes (usually contraction) largely due to isotropic conversion than non-illuminating surface .

- Endurance in fatigue with number of actuations cycles is an important factor for composite life.

- Alignment of director and domains in LC-Elastomer by mechanical stretching force (affects crosslinking) functions to control the motion and restore original nematic structure by strain energy .

- Molecular heat sources restrict utilization of UV illumination (high energy radiation) for safety motives and cost.

- Cycles of actuation graphically follow the exponential and log decay for recovery. Randomized isotropic phase of LC fails to pilot the light and hence tailor refractive index. 
- Dimensional, conc., and shape variation of nano-incorporation in LC composite can steer absorbed wavelength because of quantum confinement

\section{3D/4D Printing of LC}

Additive printing or alternatively named as 3D printing is the manufacturing tactics which has enthralled researchers ubiquitously and thrived across multitude fields since its breakthrough in 1980s [106]. In past three decades, this freshman has established its realm over conventional methodologies of manufacturing, which is attributed to its convoluted fabrication, multi-functional/material accommodation, delicate and lightweight geometries, prime resolution, engineering control over thickness and intricacy, and layered printing [107]. Development commenced with stereolithographic printing (SLA) (relied on resin photopolymerization embodying mainly acrylates [108]) and progressed to an extrusion directed filament rooted method fused deposition modeling (FDM), selective laser sintering (SLS) that involves sintering controlled powder stemmed process, selective laser melting (SLM) that involves laser melting of powder, polyjet that skillfully accommodate number of materials in printing providing a decorative hue and direct ink writing (DIW) [109-112]. In SLA, low viscosity resin layers having resolution in range of $\mu \mathrm{m}$ to $\mathrm{nm}$ are subsequently cured by UV illumination and successive stacking of layers bestows requisite $3 \mathrm{D}$ entity which is followed by post-curing. Scanning progresses line by line and output designs are governing by initiator and precursor concentrations, incident light intensity and exposure time, and further modulations are bestowed by additives [113]. In contrast, FDM employs thermoplastic solid filament which is dispensed from printer nozzle, the path of which is piloted by slicing software. FDM's rife presence is contributed by its low-cost and easy-handle nature which is 
most commonly practiced for polycarbonate, acrylo butadiene styrene, poly lactic acid, polyurethane and its composites [114,115]. Nonetheless, SLS and SLM are powder-based process whose densification is contributed by laser guide transferring energy in a nitrogen or argon atmosphere evading its contact with oxygen which initiates oxidation or other undesired reactions. Polyjet or alternatively multi-material printing renders different jets of varied material and color to impart complicated coloring effect in 3-dimension (and also gradual change in mechanical and other properties) followed by curing with UV light energy $[113,116]$. DIW is widely an extrusion-type printing whose ink formulation embodies shearthinning component which enables self-support (viscosity increases) to printed structures after being sheared through nozzle [117].

Material printability traverses across metals such as titanium (Ti) and nickel (Ni) [118], including polymers carbonate-caprolactone [119], hydrogels such as hyaluronic acid, gelatin and silk fibroin [120,121], ceramics [122] and composite materials such as carbonatehydroxyapatite [123]. Printing operation commences with a model prototype in 3D software (CAD/CAM, Solidworks, etc.) where a requisite design with dimensional specifications is recorded and synchronized with nozzle (in FDM) or image (in SLA) for printing [124]. With progress in material dispensing or laying, parameters such as printing speed, layer dimensions, orientation, layering time, shrinkage, porosity, temperature, laser depth and powder dimension are considered for effective printing [125]. Accounting all aforesaid factors, 3D printing is exploited in multifarious domains e.g. biomimetic [126,127], biomedical (tissue and drug engineering) [128,129], electronics [130], origami [131] and fashionwares [132]. However, the flourishing technology was impeded with time-consuming complex printing (hollow designs or cantilever type), support material, staticity, time carrying deformation due to 
residual stress. MIT Professor Skylar Tibbits in 2013 channelized aforesaid residual stress controlled deformation into deliberate transformation over time taking account the shape memory materials (SMM) and excited by stimulus which seeds the evolution of 4D Printing $[51,117,133]$. SMM concept was introduced in 1961 by Venon and is one category of stimuliresponsive materials, other being shape-change materials. These materials are caricatures of phenomenon evinced in biological systems like chameleon, venus flytrap, mimosa pudica plant etc. in response to certain external trigger. This stimulation transforms the functionality (shape, size, color etc.) of as-fabricated to temporary design determined by modeling. If the temporary change vanishes with stimulus itself (when stimulus is turned off) and original functionality recoups, material is shape-change material (Figure 13). If temporary shape is imprisoned after withdrawing stimulus and parent structure is reclaimed only with second actuation these are SMM (Figure 13). Thus, unveiling the ability of programming and memory in materials known as shape memory effect (SME). This SME can be one-way, twoway or multi-way relying on the temporary shapes $(1,2$, and $>2$ respectively) other than parent shape $[117,134]$. Owing to its SME, the domain is burgeoning while accommodating all possible materials from polymers to ceramics including LCs whose innate structural transition in external trigger and resultant optical attunable properties have been an inspiration for diverse applications (sensors, biomedical, actuators, LCDs etc.) [135-139]. Diversified stimuli are utilized for structural metamorphosis e.g. thermal, magnetic, light, water, current, $\mathrm{pH}$ and voltage which relied on the reinforcement characteristics and are akin to driving forces utilized for LCs [114,140,141]. These stimuli accompanied with stress steadily or anisotropically induce strain in material (based on design). For e.g. in hydro stimulus water ingress to swell hydrogels compelling to change shape, solvent and $\mathrm{pH}$ are extended cases of 
hydro stimulus by being selective to certain groups and conditions, light radiations caused photo-crosslinking which is reversible at subsequent application, thermal stimulus takes in account the mobility and entanglement of polymer chains for shape transformation at transition temperature, current and voltage stimuli indirectly induce heat through electron collision and thereby contributes to change, and magnetic stimulus transforms by indirect thermal energy or field-assisted orientation of dipoles resulting in perceivable change (Figure 14) $[134,142,143]$. Applications are multidimensional (as in $3 D$ Printing) but only the variability has stretched in time and space. Technology also inherits some loopholes for future research e.g. mass manufacturing (essential for access to consumer), scaling (discrepancy in stepping from nano-micro-macro), cycles of usability etc. [124].

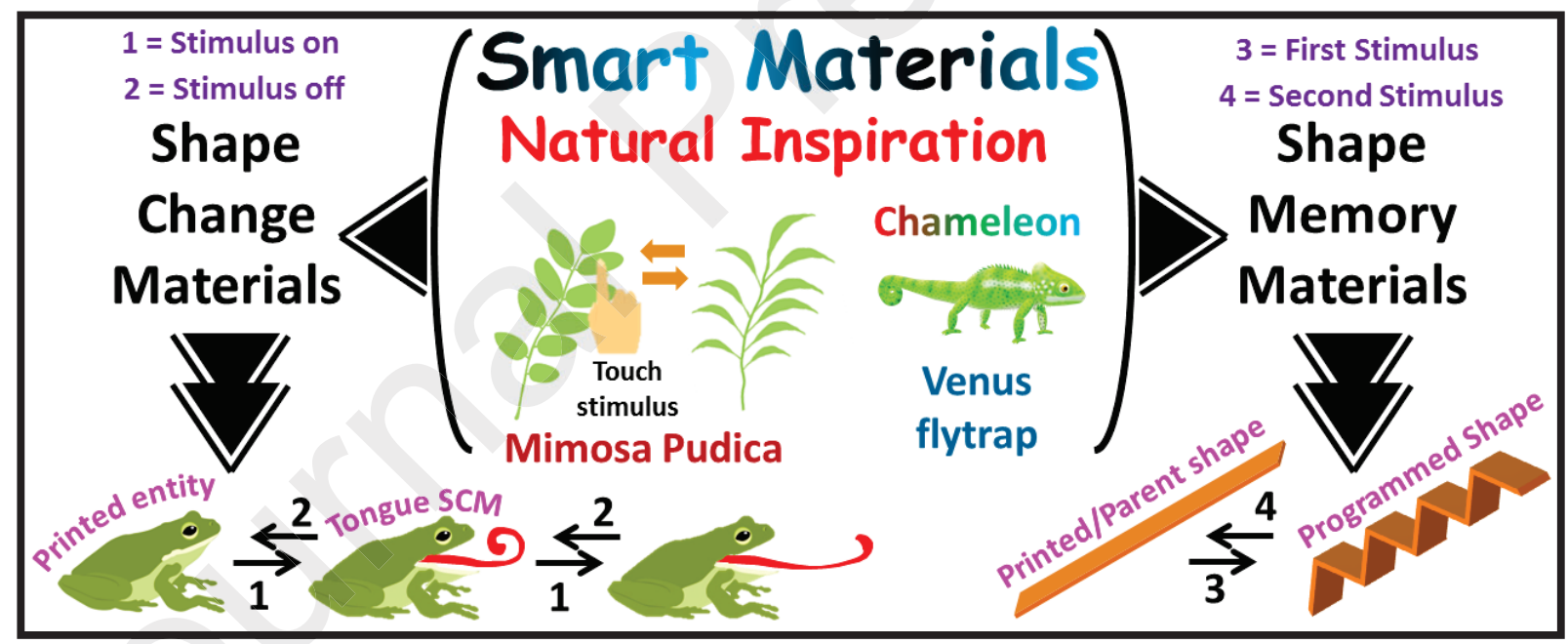

Figure 13. Classification of smart materials into SCM (changes back as stimulus is retracted) and SMM (retains shape when stimulus removed) whose process is derived from natural creatures. 


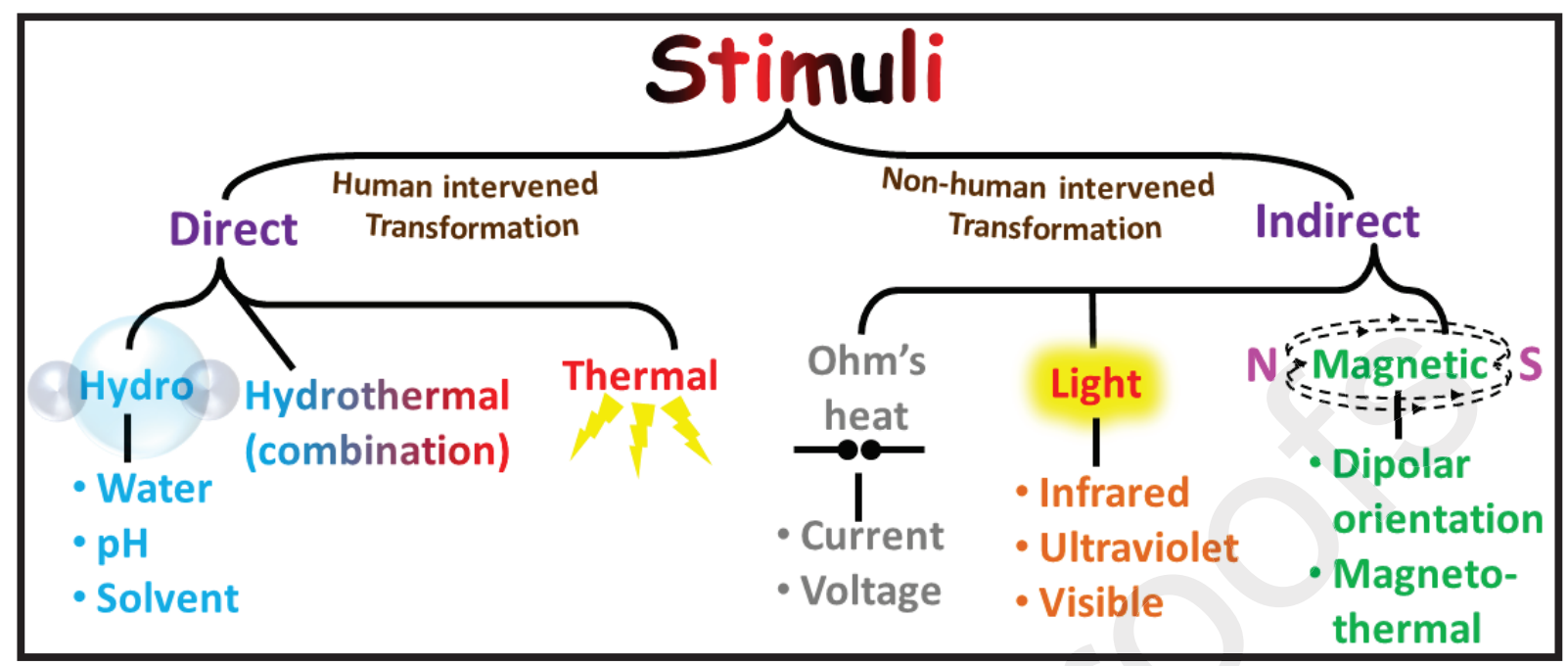

Figure 14. Categorization of applied stimuli into direct and indirect with further division into others differentiated based on mechanism.

Shape changing/memory are the entailed characteristics of LC bestowed by mesogenic arrangement in and around polymeric backbone (crosslinking) [144], combined with aroma of 3D printing ( $=4 \mathrm{D}$ printing) cherish complexity and resolution. Mesogens alignment (homeotropic or planar) (Figure 15) attune the strain production parallelly or perpendicularly to its director axis. Auguste et al. conjoined two orientations (inkjet printing of homeotropic layer over planar LC- triggered by current) which bestowed the film heterogeneity and zero Poisson's ratio within 10\% deformation conferring ubiquitous non-linear strain (irrespective of direction) thus preventing unfortunate failure of material [144]. Accompanied with aforesaid current heating, laminated 2-way reversible actuator was designed using hybrid manufacturing (LC elastomer-molding, base (Tangoblack)-polyjet, Ag ink (wire)- DIW (Figure 16)) which had been tested for 100 cycles stability. Actuation period ranged 3-10 min and similar for relaxation and was controlled by current (I) extent (1.3-2A) (time $\propto$ I). The laminate was also acclimatized to sequential actuation by administering current in multiple-set of wires (Figure 16) [145]. Unlike hybrid printing, direct dispensing of RM82 (acrylate based) LC could confer 
variable twists, cone, hemisphere, chiral and multiple intricate shapes having uniformity in properties than laminated ones. Moreover, process allowed tunable strain up to $50 \%$ when trigger temperature ranges $30^{\circ} \mathrm{C}-45^{\circ} \mathrm{C}-90^{\circ} \mathrm{C}$ while $40 \%$ (strain) when temperature ranges room-temperature $160{ }^{\circ} \mathrm{C}-200{ }^{\circ} \mathrm{C}$ under thermal condition in air or oil. Dissimilarity could be attributed to precursor UV crosslinking (its power density, time issued, and frequency (at one time or in sets) e.g. $10 \mathrm{~mW} / \mathrm{cm}^{2}$ for $5 \mathrm{~min}$ and $250 \mathrm{~mW} / \mathrm{cm}^{2}$ in two stage- $150 \mathrm{sec}$ each), heating or other environmental conditions $[49,135]$.

Additionally, these had been experimented for snap-through weight lifting (occurring in 80 milliseconds) and lens with varying focal length for variegated smart utilization in medicals $[49,135]$. Kotikian et al. had employed high-temperature DIW for printing thixotropic LC ink at $50^{\circ} \mathrm{C}$ which had expanded to $\sim 30 \%$ perpendicularly in $180 \mathrm{sec}$ but relaxation occurred in $210 \mathrm{sec}$ owing to its inappropriate cooling. Roach et al. designed variegated entities by DIW from laminated layers of acrylate and silica derived elastomer ink for thixotropic effect for UV curing, glassy ink for maintaining stiffness with temperature (inkjet printed), and thiol containing RM257 LCE for reversible stimulation under the effect of joule heating by applying current. Entropic enhancement at $42^{\circ} \mathrm{C}$ by thermal stimulus owing to LC transition (nematic $\leftrightarrow$ isotropic) resulted in $\sim 900 \%$ and $15 \%$ extension and contraction respectively. Box (4 min total actuation time), gripper (30 sec clasping and $5 \mathrm{sec}$ unclasping) (Figure 17) and hand ( $5 \mathrm{sec}$ bending time) designs were printed and actuated at $1.25 \mathrm{~A}, 1.5 \mathrm{~A}$ and $1.5 \mathrm{~A}$ respectively presenting anisotropic bending which could optimize phonons band gap for optical switches, soft-robotics, medical devices, and actuators [146]. Similarly, Saed et al. had demostrated DIW-printed RM257 (being a low molecular weight compound than RM82) subsided temperature for nematic to isotropic change such that when LC had RM82:RM252 = 
100:0, temperature was $\sim 41^{\circ} \mathrm{C}$ while it dropped down to $\sim 12^{\circ} \mathrm{C}$ for $25: 75$ and finally, isotropic conformation at 0:100 ratio. This (T-transition) was in contrast to aforementioned study with laminated structures (which was higher). Authors, by tuning their formulation ratio (100:0 to 0:100), demonstrated sequential actuation (Figure 18) under thermal energy with speedy strain recovery at higher temperature $\left(110^{\circ} \mathrm{C}\right)$ for proposed operations in grippers (soft actuators) and body-temperature workable devices [14]. For the mentioned studies [144,147], nozzles from extrusion printers had fulfilled an auxiliary function of aligning mesophase in the direction of stress predicating complex transmuting freedom. Studart's group realistically played with temperature in stressed/strained LC stream from nozzle to fabricate core-shell within same material. Hot stream flowing out solidified on its outer in aligned nematic and resultant gradient (high-temperature) in interior rendered thermally driven random alignment (Figure 19). Furthermore, FDM printed LC-Polymer extrudate ushered improved mechanical properties (modulus, strength, stiffness, toughness) and the resultant could be recycled in view with environmental concern [32].

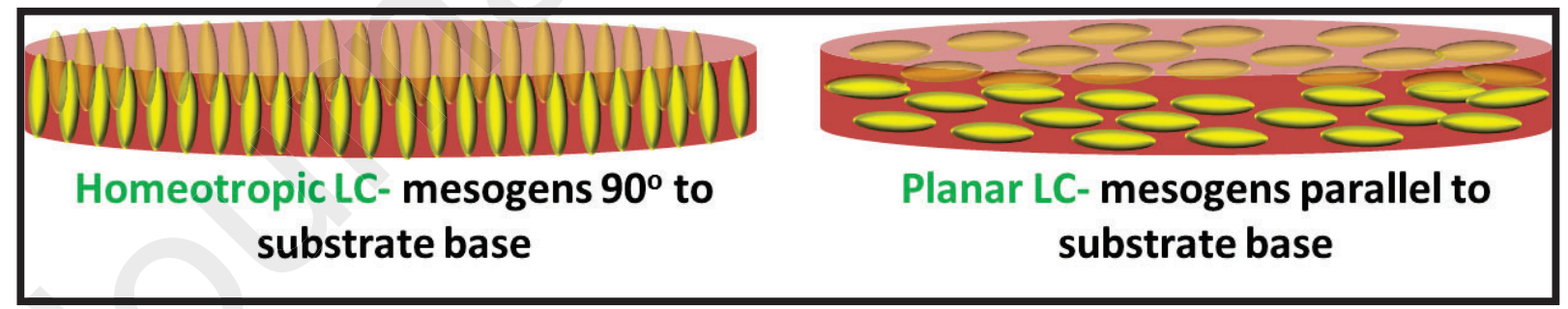

Figure 15. Systematic depiction of homeotropic and planar LC. 


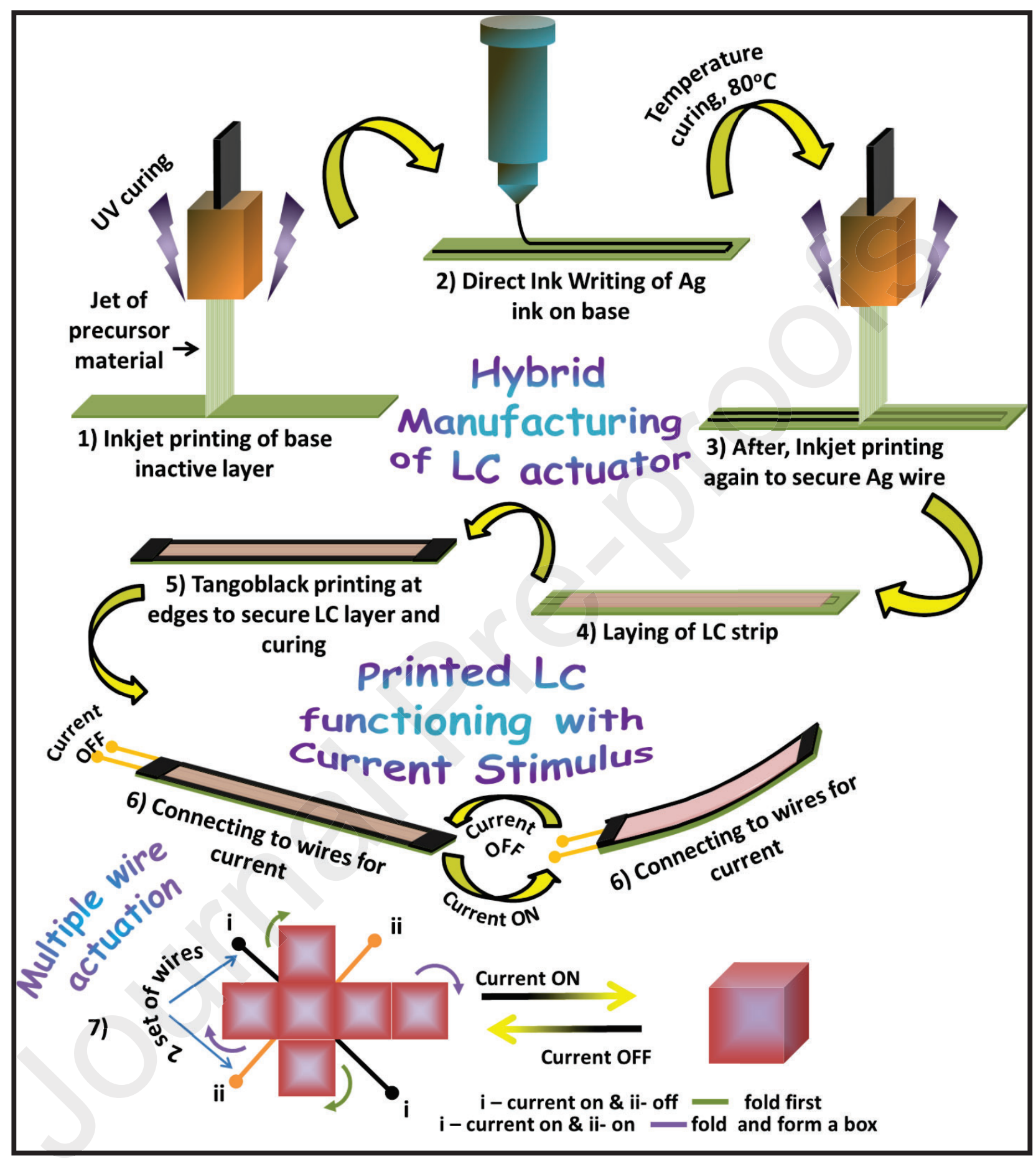

Figure 16.Depiction of hybrid manufacturing of LC laminates and multi-wire current actuation. 


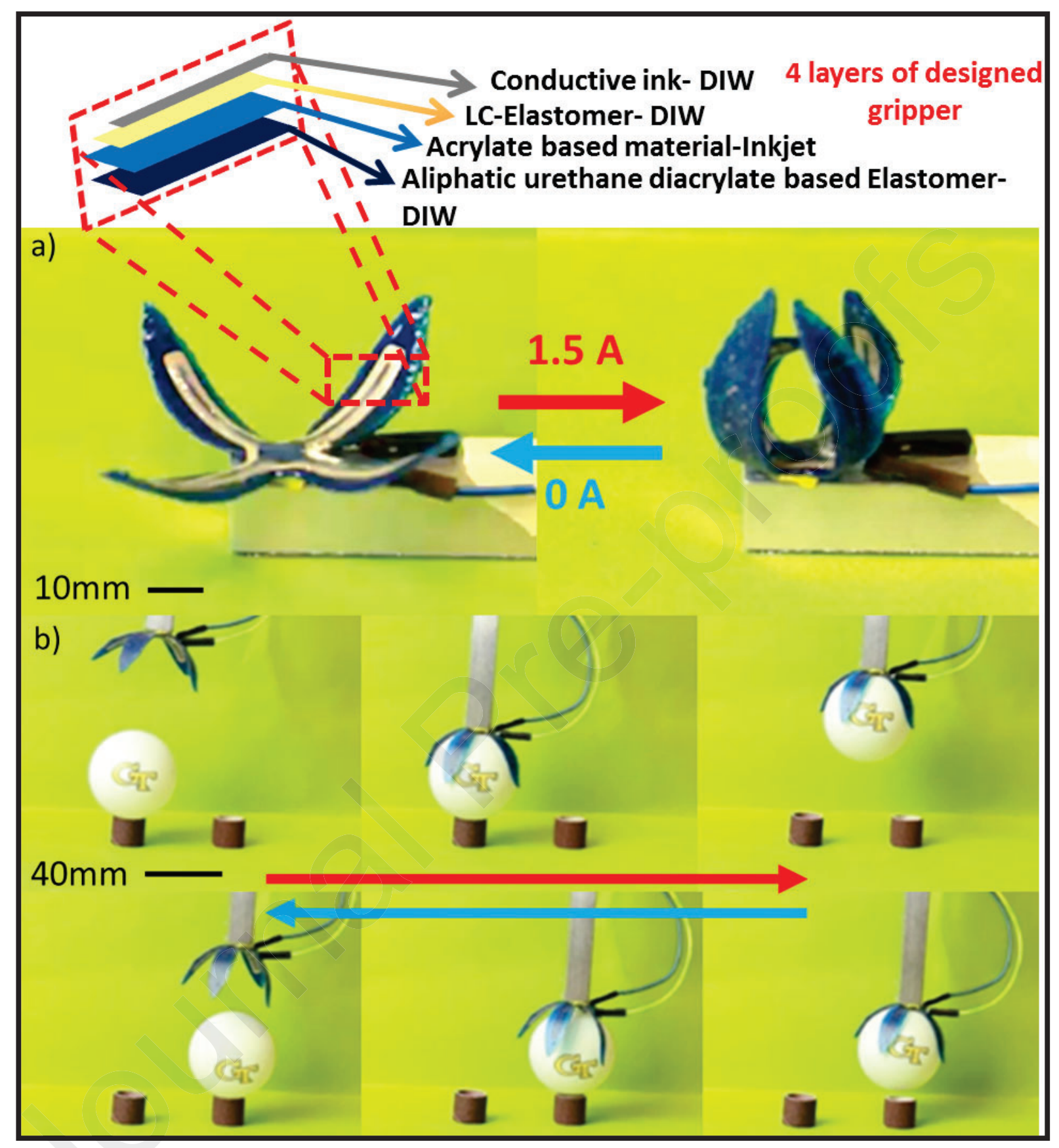

Figure 17. Describes a) 3D printing of gripper in layering perspective (4 layers of printing) of LCE, acrylates (UV crosslinking), and ink for conduction (1.5 A) empowering joule -steered transformation. b) Stages traversed in grasping ball while current is turned on (30 sec for grasping) and off (5 sec releasing) [146]. 


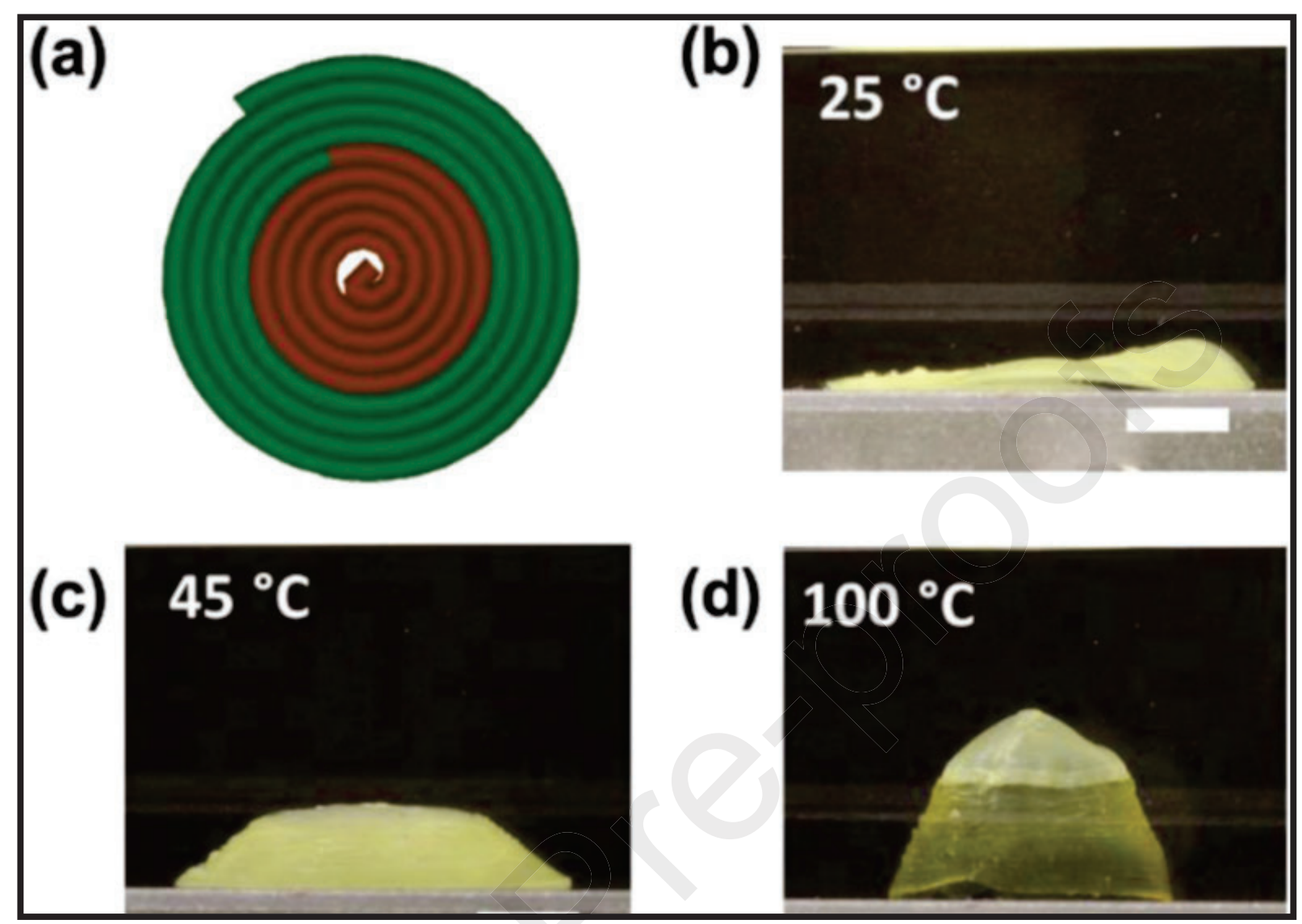

Figure 18. Depiction of chronological actuation in printed LC where a) designed prototype in software in which outer green and inner red color had low- and high- temperature LCElastomer respectively aimed at bequeathing order of structure evolution, b) room temperature $\left(25^{\circ} \mathrm{C}\right)$ printed LC using DIW with azimuthal defect $(+1$ topology), c) actuation of outer layer at $45^{\circ} \mathrm{C}$ and d) inner layer at $100^{\circ} \mathrm{C}$ owing to as-defined design criteria [14]. 


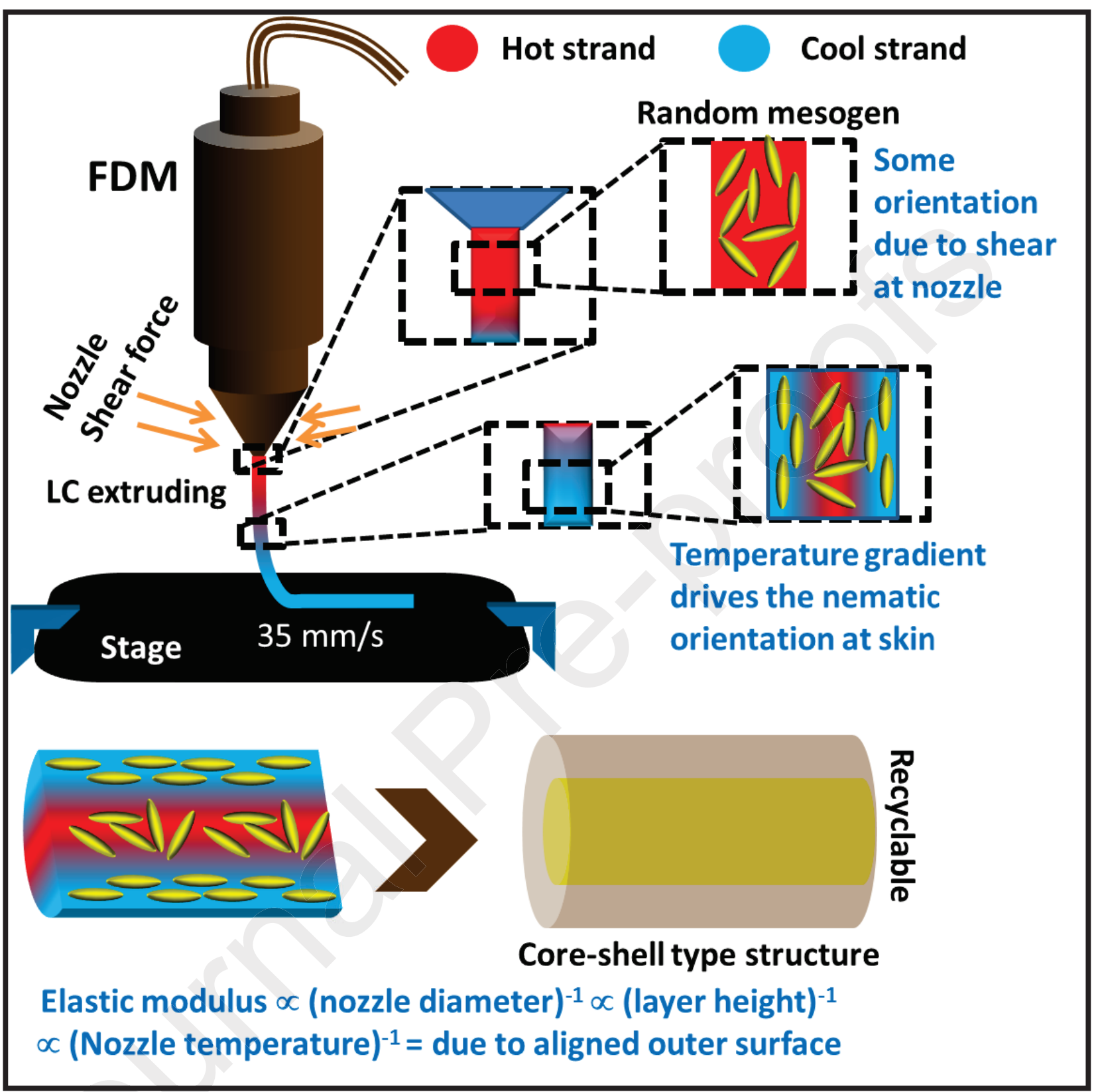

Figure 19.Core-shell production of LC by orientational gradient during cooling.

With above said literature for LC $[49,50,135,144,145,147]$, authors have reported thermal or non-interfered actuation (current) and the trio of $\mathrm{LC}+3 \mathrm{D}$ printing + photothermal are still in the offering with the world's curious minds and hence scope for delving in materials details, process and other prerequisites remain.

5. Biomedical Applications of Photothermal LCs 
This section succinctly delineates biomedical applications based on photothermal actuation with LCs (Figure 20). Nature is always a big-bang of curiosities which also encases LCs, for example, camouflaging and iridescence functioning in insects, beetles, and other creatures which are due to chitin presence signifies cholesteric LC [3]. Not constraining to these, lyotropic LC characteristics had been unfolded in DNA regimes which were doped with Au$\mathrm{Ag}$ (dimension $<2 \mathrm{~nm}$ ) and were perceived as suitable in optical biomarkers in-vivo and invitro [96]. Huang et al. also mimicked lyotropic LC in GO (in $1.6 \mathrm{mg} / \mathrm{ml}$ ) assisted with the dispersion of sodium alginate (by ultrasonication [148]) for self-assembly (concentration for LC reduced to $0.2 \mathrm{mg} / \mathrm{ml}$ ascribed to hydrogen bonding between $\mathrm{GO}$ and sodium alginate) which had promoted cytocompatibility and proliferation of cells for tissue engineering [149]. Yeo et al. devised a non-drug treatment of scars (hypertrophic scar and keloid scar, these are the painful after-effect of wound treatment) with microneedles (LCP-Vectra) covered base, proficiently avoiding contact with dermis skin and thus attenuating pain.

Further, keloid scar treatment demonstrated $\sim 84 \%$ cell apoptosis in 12 hours and $66.7 \%$ efficacy in hypertrophic scars [150]. However, photo-actuation (UV) was discerned by Dmitrenko et al. in previtamin-D with ordered LC phases (appertained to nematic $\rightarrow$ isotropic conversion) [151]. Employing thermal stimulus, He et al. installed RM257 LC-elastomer on skeleton to demonstrate mimicking of artificial muscles (jaw, hand and leg movement) in temperature range $5-95^{\circ} \mathrm{C}$ through contraction $(40 \%$ in $30 \mathrm{sec}$ )-expansion (relax back in 15 sec) [152]. In contrast to UV, photothermal is the proclivity of light to induce heat for carrying out mutation in microstructure and functionality in human perceivable dimension. Tian et al. had also manifested muscle actuation with polydopamine containing LC-elastomer to bestow photothermal actuation in near-IR mimicking mammal muscle stress magnitude within 0.5 
sec. The researchers also observed proportionality of near-IR illumination time with induced stress, temperature amplification, and pre-strain meanwhile, displaying $1 \mathrm{~mm} / \mathrm{sec}$ speed of designed fish with instant response in $1 \mathrm{sec}$ [42]. For medication in another biomedical domain, Thapa et al. had encased polyethylene glycol (PEG)-LC (contains lipid) with graphene oxide (GO) and was engineered with docetaxel toxoid for dual functions of targeted drug transfer and hyperthermia causing cancer cells death [39]. Analogously, Luo et al. had demonstrated $\mathrm{pH}$ and photothermal treatment of tumor and by integrating $\mathrm{Au}$-nanoshells with liposomes and releasing Oleanolic acid for tumor medication in near-IR [44].

In short, the literature of LC and medication parallels peanut in prospect with biomedical exploration and performance; especially with photothermal non-contact metamorphosis, thereby, necessitating meticulous efforts. 


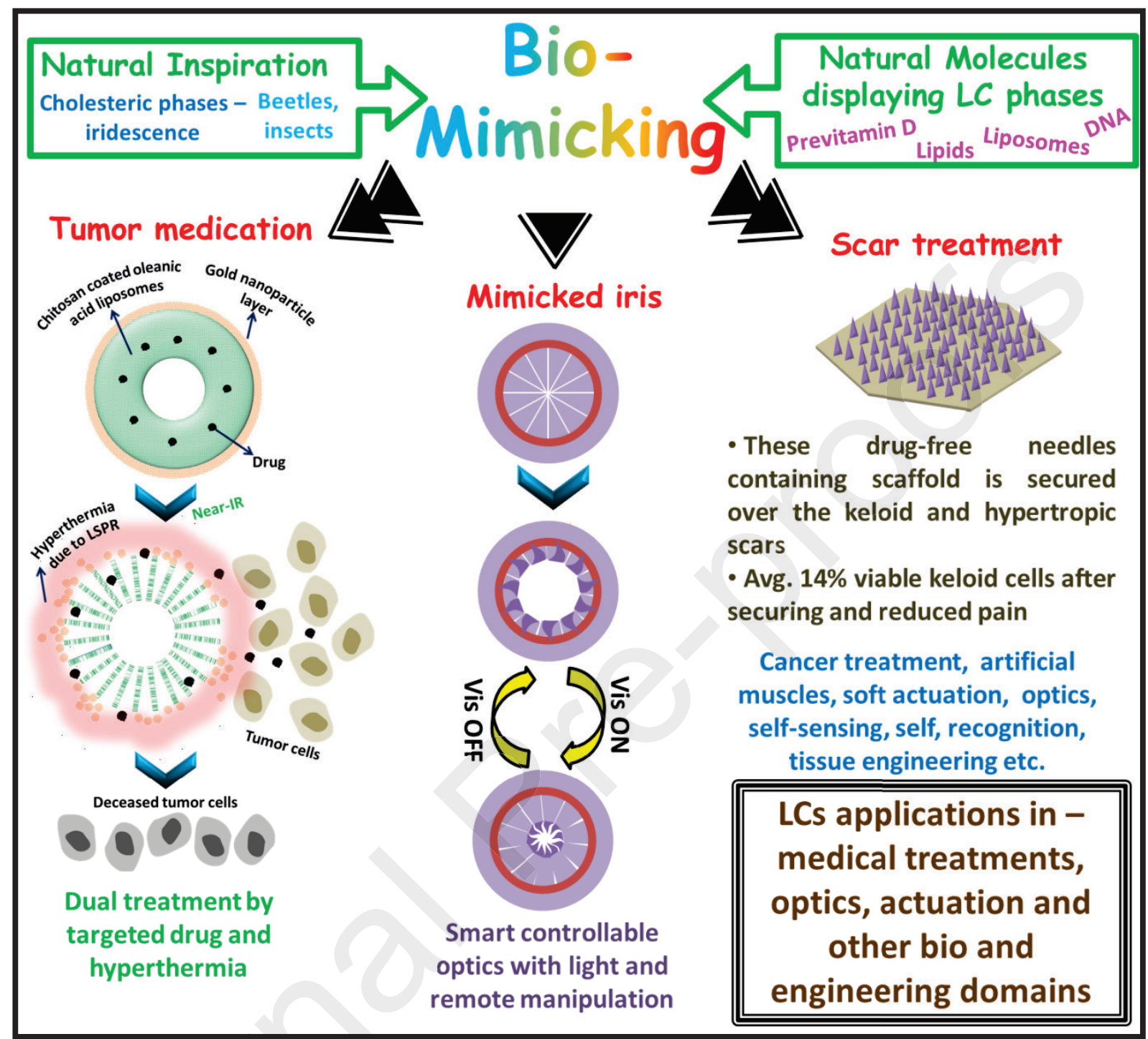

Figure 20. Summarized biomedical application of LC.

\section{Key Features, Future, and Conclusion}

LC-polymers (un-cross-linked) showcase retardancy to flames with high-temperature usability, resistance to chemicals, weathering, and permeability. However, mechanical achievement can be enhanced with mild or ample crosslinking which encourages stability and administer shape memory but captivate its dynamicity. Slow stimuli response of LC-elastomer can be amplified with photothermal additives (focusing on photothermal) e.g. CNT, graphene 
(challenged by their dispersibility), Au, Ag (aid crosslinking), dyes, etc. which can lessen response time to $\sim 1 \mathrm{sec}$. Moreover, their instability (agglomeration) in LC confronts nonuniform stimulation (disturbance) and qualitative adhesion thereby, requiring quantitative optimization (dispersibility and concentration). In addition, geometry and dimensions of additives are also the key to healthy trigger e.g. larger metal-NP suppresses photothermal quality, Au-nanorods displays efficacy over shells in energy (heat) production, fibers etc. Besides, the most onerous task is the mesogen alignment (monodomain), stimulation fatigue on polymer chain, and LC's macro-dimensional mimicking. With respect to light trigger, low energy near-IR/Vis responsive LC are suitable for cells and tissues owing to their transparency in that region preventing damage $[18,20,80,93,145,153]$.

Additives like CNTs, gold and nanoparticles plays paramount importance in administering material properties but their agglomeration negatively influences isotropic transformation or localized hyperthermia temperature which may lead to degradation if increases uncontrollably [86]. Though LCs capability in being able to display 2-way actuation has profound significance in actuators, sensors, grippers and other biomedical fields with variegated stimuli but it also confronts challenges which seeds for future research potential. Pre-eminent adversity of LC lies in its commercialization in multidisciplinary fields of engineering and in medicinal treatments. This is further followed by purity requirement of precursors, processing time efficiency, and chiefly by bulk manufacturing along with cost (in range of 2 USD). Literature reveals that LC DIW printing against gravity (along height) in 3D is dimensionally barricaded which can be overcome by formulating oligomers for rapid self-strengthening of thixotropic filament after ink extrusion. Futuristic design convolution necessitates study in detail based on relation of mesogen interaction with polymeric chain 
relaxation taking in account the physics of working of LC-Elastomers [154]. Though 3D/4D printing circumscribes convoluted and hollow design with gradient effect in color and properties but obstruction occurs in emulation of polymer state with that required 3D printing process. In addition, burgeoning in recyclability, healing and welding can aid in recovery of entity damage or modification requirement at final stage truncating in wastage and production cost. Yet another feature that necessitates extensive research is fine-tuning the device e.g. robots for environmental extremity for unpredictable conditions when targeting space applications, Arctic Circle, in harsh weathers (snow, rain etc.) or sever altitude, hightemperature stability $[87,88]$. Invent of 4D printing (SME of SMM in its core) has instigated a different perspective and design freedom for their remedy with its shape-responsiveness or functionality change but its infancy stage and material selection entails more meticulous endeavors from various disciplines across globe.

Collectively, aforesaid features have impelled scientists for multitude variations in experimentation for multifarious applications. For instance, bio-imitated flytraps with remote grabbing, solar device opting heliotropism for garnering energy, emulating caterpillar movement (crawl, climb etc.), mimicking swimmer with LC, micro-robots, artificial muscles, aerospace devices equipped with deployability with low temperature working allowance, window switch-ability from a distant and laminated multifunctional actuator (soft machine). Furthermore, in medication, fabricating interactive 3D scaffolds with induced porosity for cell proliferation, extracellular matrix growth along with added features of biodegradability, recyclability, biocompatibility, for tissue engineering and drug delivery regimes $[53,74,75]$. The plausibility of LC with printing can further be acknowledged by its market economy 
which escalates to achieve $\sim 537$ million USD for 4D manufacturing and 1.2 billion USD for LC-polymers $[2,155]$ thus can be envisaged to plentiful application expansion.

In this regard, present review fathoms the chemistry basics of photothermal metamorphosis in LC polymers and elastomers (acquire just-adequate crosslinking) by reinforcements. Delocalization of electrons, its transferability to higher energy conducting band, its nonradiative relaxing (in dyes, CNT, Graphene, etc.) and LSPR in nano-dimensional metalparticles confer thermal conversion from incident photons. Acquiring the photothermal ability, review discusses the LC with respect to various additions as CNT, azobenzene, graphene, Ag, aniline, and $\mathrm{Au}$, while acknowledging their structural, dimensional, and functional variations along with associated performances. Review further appraises the advanced fabrication with 3D additive manufacturing of $\mathrm{LC}$ re-entitling as 4D printing. Biological mimicking, its exploration in treatments is the dire need which with LC collaborations have facilitated meticulous medication. Finally, the review concludes while traversing across characteristics and potential of LC in medicals, sensing, actuators and others which are in the offing.

\section{Acknowledgment}

Authors are thankful to Dr. CP Ramanarayanan, Vice Chancellor, Defence Institute of Advanced Technology (DU), Pune for the support. Prasansha Rastogi would also like to acknowledge Mr. Prakash Gore and Mr. Swaroop Gharde for their persistent technical support during the writing stage.

\section{References}

[1] Grand View Research, Liquid Crystal Polymers (LCP) Market Analysis By Application (Electrical \& Electronics, Industrial Machinery, Automotive) And Segment Forecasts 
To 2020, (2015). https://www.grandviewresearch.com/industry-analysis/liquid-crystalpolymers-market (accessed November 23, 2018).

[2] MarketsandMarkets, Liquid Crystal Polymer Market worth 1.2 Billion USD by 2020, (n.d.). https://www.marketsandmarkets.com/PressReleases/liquid-crystal-polymerresin.asp (accessed November 23, 2018).

[3] D. Martella, C. Parmeggiani, Advances in Cell Scaffolds for Tissue Engineering: The Value of Liquid Crystalline Elastomers, Chem. - Eur. J. 24 (2018) 12206-12220. doi:10.1002/chem.201800477.

[4] D. Iqbal, M. Samiullah, Photo-Responsive Shape-Memory and Shape-Changing Liquid-Crystal Polymer Networks, Materials. 6 (2013) 116-142. doi:10.3390/ma6010116.

[5] C.H. Chan, C.H. Chia, S. Thomas, Physical chemistry of macromolecules macro to nanoscales, 2014. http://www.crcnetbase.com/isbn/9781926895642 (accessed June 9, 2019).

[6] H.K. Bisoyi, Q. Li, Light-Driven Liquid Crystalline Materials: From Photo-Induced Phase Transitions and Property Modulations to Applications, Chem. Rev. 116 (2016) 15089-15166. doi:10.1021/acs.chemrev.6b00415.

[7] Q. Li, Photoactive functional soft materials: preparation, properties, and applications, 2019.

[8] D.L. Thomsen, P. Keller, J. Naciri, R. Pink, H. Jeon, D. Shenoy, B.R. Ratna, Liquid Crystal Elastomers with Mechanical Properties of a Muscle, Macromolecules. 34 (2001) 5868-5875. doi:10.1021/ma001639q.

[9] P. Beyer, M. Krueger, F. Giesselmann, R. Zentel, Photoresponsive Ferroelectric LiquidCrystalline Polymers, Adv. Funct. Mater. 17 (2007) 109-114. doi:10.1002/adfm.200600513.

[10] C. Ohm, M. Brehmer, R. Zentel, Liquid Crystalline Elastomers as Actuators and Sensors, Adv. Mater. 22 (2010) 3366-3387. doi:10.1002/adma.200904059.

[11] E.B. Priestley, P.J. Wojtowicz, P. Sheng, Introduction to Liquid Crystals, Springer US, Boston, MA, 1976. http://dx.doi.org/10.1007/978-1-4684-2175-0 (accessed May 27, 2019).

[12] J.M. Boothby, J. Samuel, T.H. Ware, Molecularly-ordered hydrogels with controllable, anisotropic stimulus response, Soft Matter. (2019) 10.1039.C9SM00763F. doi:10.1039/C9SM00763F.

[13] B.T. Michal, B.M. McKenzie, S.E. Felder, S.J. Rowan, Metallo-, Thermo-, and Photoresponsive Shape Memory and Actuating Liquid Crystalline Elastomers, Macromolecules. 48 (2015) 3239-3246. doi:10.1021/acs.macromol.5b00646.

[14] M.O. Saed, C.P. Ambulo, H. Kim, R. De, V. Raval, K. Searles, D.A. Siddiqui, J.M.O. Cue, M.C. Stefan, M.R. Shankar, T.H. Ware, Molecularly-Engineered, 4D-Printed Liquid Crystal Elastomer Actuators, Adv. Funct. Mater. 29 (2019) 1806412. doi:10.1002/adfm.201806412.

[15] A.K. Bhowmick, ed., Current topics in elastomers research, CRC Press, Boca Raton, FL, 2008.

[16] P.J. Collings, M. Hird, Introduction to liquid crystals: chemistry and physics, Taylor \& Francis, London, $1997 . \quad$ http://ezproxy.standrews.ac.uk/login?url=http://www.myilibrary.com?id=40253 (accessed May 27, 2019). 
[17] X.-H. Han, X.-W. Yang, S. Chen, H. Luo, D. Zhang, H.-L. Zhang, Multiple Effects Tailoring the Self-organization Behaviors of Triphenylene Side-chain Liquid Crystalline Polymers via Changing the Spacer Length, Chin. J. Polym. Sci. 36 (2018) 960-969. doi:10.1007/s10118-018-2108-9.

[18] T. Kato, J. Uchida, T. Ichikawa, B. Soberats, Functional liquid-crystalline polymers and supramolecular liquid crystals, Polym. J. 50 (2018) 149-166. doi:10.1038/pj.2017.55.

[19] T.J. White, Photomechanical effects in liquid crystalline polymer networks and elastomers, J. Polym. Sci. Part B Polym. Phys. 56 (2018) 695-705. doi:10.1002/polb.24576.

[20] T.J. White, D.J. Broer, Programmable and adaptive mechanics with liquid crystal polymer networks and elastomers, Nat. Mater. 14 (2015) 1087-1098. doi:10.1038/nmat4433.

[21] A. Zabara, R. Mezzenga, Controlling molecular transport and sustained drug release in lipid-based liquid crystalline mesophases, J. Controlled Release. 188 (2014) 31-43. doi:10.1016/j.jconrel.2014.05.052.

[22] H. Zeng, O.M. Wani, P. Wasylczyk, R. Kaczmarek, A. Priimagi, Self-Regulating Iris Based on Light-Actuated Liquid Crystal Elastomer, Adv. Mater. 29 (2017) 1701814. doi:10.1002/adma.201701814.

[23] M. Lavrič, V. Tzitzios, G. Cordoyiannis, S. Kralj, G. Nounesis, I. Lelidis, Z. Kutnjak, Blue Phase Range Widening Induced by Laponite Nanoplatelets in the Chiral Liquid Crystal CE8, Mol. Cryst. Liq. Cryst. 615 (2015) 14-18. doi:10.1080/15421406.2015.1066554.

[24] M. Lavrič, V. Tzitzios, S. Kralj, G. Cordoyiannis, I. Lelidis, G. Nounesis, V. Georgakilas, H. Amenitsch, A. Zidanšek, Z. Kutnjak, The effect of graphene on liquidcrystalline blue phases, Appl. Phys. Lett. 103 (2013) 143116. doi:10.1063/1.4824424.

[25] C. Zou, J. Sun, M. Wang, J. Wang, Y. Wu, L. Zhang, Z. Zhu, G. Xiong, L. Jiang, T. Ikeda, H. Yang, A UV-Responsive Multifunctional Photoelectric Device Based on Discotic Columnar Nanostructures and Molecular Motors, Adv. Mater. 31 (2019) 1806016. doi:10.1002/adma.201806016.

[26] M. Trček, G. Cordoyiannis, B. Rožič, V. Tzitzios, G. Nounesis, S. Kralj, I. Lelidis, E. Lacaze, H. Amenitsch, Z. Kutnjak, Twist-grain boundary phase induced by $\mathrm{Au}$ nanoparticles in a chiral liquid crystal host, Liq. Cryst. 44 (2017) 1575-1581. doi:10.1080/02678292.2017.1306887.

[27] T. Sasaki, T. Ikeda, K. Ichimura, Photochemical control of properties of ferroelectric liquid crystals. Photochemical flip of polarization, J. Am. Chem. Soc. 116 (1994) 625628. doi:10.1021/ja00081a024.

[28] T. Ikeda, T. Sasaki, K. Ichimura, Photochemical switching of polarization in ferroelectric liquid-crystal films, Nature. 361 (1993) 428-430. doi:10.1038/361428a0.

[29] C. Zou, J. Wang, M. Wang, Y. Wu, K. Gu, Z. Shen, G. Xiong, H. Yang, L. Jiang, T. Ikeda, Patterning of Discotic Liquid Crystals with Tunable Molecular Orientation for Electronic Applications, Small. 14 (2018) 1800557. doi:10.1002/smll.201800557.

[30] H. Yang, J.-J. Liu, Z.-F. Wang, L.-X. Guo, P. Keller, B.-P. Lin, Y. Sun, X.-Q. Zhang, Near-infrared-responsive gold nanorod/liquid crystalline elastomer composites prepared by sequential thiol-click chemistry, Chem. Commun. 51 (2015) 12126-12129. doi:10.1039/C5CC02599K. 
[31] G. Vantomme, A.H. Gelebart, D.J. Broer, E.W. Meijer, Self-sustained actuation from heat dissipation in liquid crystal polymer networks, J. Polym. Sci. Part Polym. Chem. 56 (2018) 1331-1336. doi:10.1002/pola.29032.

[32] S. Gantenbein, K. Masania, W. Woigk, J.P.W. Sesseg, T.A. Tervoort, A.R. Studart, Three-dimensional printing of hierarchical liquid-crystal-polymer structures, Nature. 561 (2018) 226-230. doi:10.1038/s41586-018-0474-7.

[33] C. Balagna, M. Irfan, S. Perero, M. Miola, G. Maina, M. Crosera, D. Santella, A. Simone, M. Ferraris, Antibacterial nanostructured composite coating on high performance Vectran ${ }^{\mathrm{TM}}$ fabric for aerospace structures, Surf. Coat. Technol. 373 (2019) 47-55. doi:10.1016/j.surfcoat.2019.05.076.

[34] R. Bao, M. Pan, J.J. Qiu, H.Q. Tang, C.M. Liu, Photo-polymerization of liquid crystalline monomer in oriented liquid crystal phase, Chin. Chem. Lett. 21 (2010) 1330-1333. doi:10.1016/j.cclet.2010.05.010.

[35] L. Tao, M.-L. Li, K.-P. Yang, Y. Guan, P. Wang, Z. Shen, H.-L. Xie, Color-Tunable and Stimulus-Responsive Luminescent Liquid Crystalline Polymers Fabricated by Hydrogen Bonding, ACS Appl. Mater. Interfaces. 11 (2019) 15051-15059. doi:10.1021/acsami.9b01476.

[36] J.M. Boothby, T.H. Ware, Dual-responsive, shape-switching bilayers enabled by liquid crystal elastomers, Soft Matter. 13 (2017) 4349-4356. doi:10.1039/C7SM00541E.

[37] H. Kim, J.M. Boothby, S. Ramachandran, C.D. Lee, T.H. Ware, Tough, ShapeChanging Materials: Crystallized Liquid Crystal Elastomers, Macromolecules. 50 (2017) 4267-4275. doi:10.1021/acs.macromol.7b00567.

[38] R. Yang, Y. Zhao, Non-Uniform Optical Inscription of Actuation Domains in a Liquid Crystal Polymer of Uniaxial Orientation: An Approach to Complex and Programmable Shape Changes, Angew. Chem. 129 (2017) 14390-14394. doi:10.1002/ange.201709528.

[39] R.K. Thapa, Y.S. Youn, J.-H. Jeong, H.-G. Choi, C.S. Yong, J.O. Kim, Graphene oxide-wrapped PEGylated liquid crystalline nanoparticles for effective chemophotothermal therapy of metastatic prostate cancer cells, Colloids Surf. B Biointerfaces. 143 (2016) 271-277. doi:10.1016/j.colsurfb.2016.03.045.

[40] M. Bi, Y. Shao, Y. Wang, J. Zhang, H. Niu, Y. Gao, B. Wang, C. Li, Liquid crystalline elastomer doped with silver nanoparticles: Fabrication and nonlinear absorption properties, Mol. Cryst. Liq. Cryst. 652 (2017) 41-50. doi:10.1080/15421406.2017.1357420.

[41] Z. Cheng, T. Wang, X. Li, Y. Zhang, H. Yu, NIR-Vis-UV Light-Responsive Actuator Films of Polymer-Dispersed Liquid Crystal/Graphene Oxide Nanocomposites, ACS Appl. Mater. Interfaces. 7 (2015) 27494-27501. doi:10.1021/acsami.5b09676.

[42] H. Tian, Z. Wang, Y. Chen, J. Shao, T. Gao, S. Cai, Polydopamine-Coated Main-Chain Liquid Crystal Elastomer as Optically Driven Artificial Muscle, ACS Appl. Mater. Interfaces. 10 (2018) 8307-8316. doi:10.1021/acsami.8b00639.

[43] G.C. Lama, P. Cerruti, M. Lavorgna, C. Carfagna, V. Ambrogi, G. Gentile, Controlled Actuation of a Carbon Nanotube/Epoxy Shape-Memory Liquid Crystalline Elastomer, J. Phys. Chem. C. 120 (2016) 24417-24426. doi:10.1021/acs.jpcc.6b06550.

[44] L. Luo, Y. Bian, Y. Liu, X. Zhang, M. Wang, S. Xing, L. Li, D. Gao, Combined Near Infrared Photothermal Therapy and Chemotherapy Using Gold Nanoshells Coated 
Liposomes to Enhance Antitumor Effect, Small. 12 (2016) 4103-4112. doi:10.1002/smll.201503961.

[45] L. Zhang, W. Zhang, N. Zhou, J. Zhu, Z. Zhang, Z. Cheng, X. Zhu, Preparation and Characterization of Linear and Miktoarm Star Side-Chain Liquid Crystalline block Copolymers with $p$-Methoxyazobenzene Moieties via a Combination of ATRP and ROP, J. Macromol. Sci. Part A. 46 (2009) 876-885. doi:10.1080/10601320903078164.

[46] C. Li, Y. Liu, C. Lo, H. Jiang, Reversible white-light actuation of carbon nanotube incorporated liquid crystalline elastomer nanocomposites, Soft Matter. 7 (2011) 7511. doi:10.1039/c1sm05776f.

[47] Y. Yu, T. Ikeda, Soft Actuators Based on Liquid-Crystalline Elastomers, Angew. Chem. Int. Ed. 45 (2006) 5416-5418. doi:10.1002/anie.200601760.

[48] H. Yang, G. Ye, X. Wang, P. Keller, Micron-sized liquid crystalline elastomer actuators, Soft Matter. 7 (2011) 815-823. doi:10.1039/C0SM00734J.

[49] M. López-Valdeolivas, D. Liu, D.J. Broer, C. Sánchez-Somolinos, 4D Printed Actuators with Soft-Robotic Functions, Macromol. Rapid Commun. 39 (2018) 1700710. doi:10.1002/marc.201700710.

[50] A. Kotikian, R.L. Truby, J.W. Boley, T.J. White, J.A. Lewis, 3D Printing of Liquid Crystal Elastomeric Actuators with Spatially Programed Nematic Order, Adv. Mater. 30 (2018) 1706164. doi:10.1002/adma.201706164.

[51] S. Tibbits, 4D Printing: Multi-Material Shape Change, Archit. Des. 84 (2014) 116-121. doi:10.1002/ad.1710.

[52] B. Ni, H.-L. Xie, J. Tang, H.-L. Zhang, E.-Q. Chen, A self-healing photoinduceddeformable material fabricated by liquid crystalline elastomers using multivalent hydrogen bonds as cross-linkers, Chem. Commun. 52 (2016) 10257-10260. doi:10.1039/C6CC04199J.

[53] A. Sharma, A. Neshat, C.J. Mahnen, A. d. Nielsen, J. Snyder, T.L. Stankovich, B.G. Daum, E.M. LaSpina, G. Beltrano, Y. Gao, S. Li, B.-W. Park, R.J. Clements, E.J. Freeman, C. Malcuit, J.A. McDonough, L.T.J. Korley, T. Hegmann, E. Hegmann, Biocompatible, Biodegradable and Porous Liquid Crystal Elastomer Scaffolds for Spatial Cell Cultures: Biocompatible, Biodegradable and Porous LC Elastomers, Macromol. Biosci. 15 (2015) 200-214. doi:10.1002/mabi.201400325.

[54] L.-X. Guo, M.-H. Liu, S.M. Sayed, B.-P. Lin, P. Keller, X.-Q. Zhang, Y. Sun, H. Yang, A calamitic mesogenic near-infrared absorbing croconaine dye/liquid crystalline elastomer composite, Chem. Sci. 7 (2016) 4400-4406. doi:10.1039/C6SC00758A.

[55] H.M.D. Bandara, S.C. Burdette, Photoisomerization in different classes of azobenzene, Chem Soc Rev. 41 (2012) 1809-1825. doi:10.1039/C1CS15179G.

[56] G.S. Kumar, D.C. Neckers, Photochemistry of azobenzene-containing polymers, Chem. Rev. 89 (1989) 1915-1925. doi:10.1021/cr00098a012.

[57] H. Rau, Photoisomerization of Azobenzenes, in: Photoreact. Org. Thin Films, Elsevier, 2002: pp. 3-47. doi:10.1016/B978-012635490-4/50002-0.

[58] R.R. Avirah, K. Jyothish, D. Ramaiah, Infrared Absorbing Croconaine Dyes: Synthesis and Metal Ion Binding Properties, J. Org. Chem. 73 (2008) 274-279. doi:10.1021/jo702209a.

[59] E.E. Havinga, W. ten Hoeve, H. Wynberg, Alternate donor-acceptor small-band-gap semiconducting polymers; Polysquaraines and polycroconaines, Synth. Met. 55 (1993) 299-306. doi:10.1016/0379-6779(93)90949-W. 
[60] G.T. Spence, G.V. Hartland, B.D. Smith, Activated photothermal heating using croconaine dyes, Chem. Sci. 4 (2013) 4240. doi:10.1039/c3sc51978c.

[61] N.S. Abadeer, C.J. Murphy, Recent Progress in Cancer Thermal Therapy Using Gold Nanoparticles, J. Phys. Chem. C. $120 \quad$ (2016) 4691-4716. doi:10.1021/acs.jpcc.5b11232.

[62] X. Huang, M.A. El-Sayed, Plasmonic photo-thermal therapy (PPTT), Alex. J. Med. 47 (2011) 1-9. doi:10.1016/j.ajme.2011.01.001.

[63] S. Son, X. Bai, S. Lee, Inorganic hollow nanoparticles and nanotubes in nanomedicinePart 2: Imaging, diagnostic, and therapeutic applications, Drug Discov. Today. 12 (2007) 657-663. doi:10.1016/j.drudis.2007.06.012.

[64] K.P. Unnikrishnan, V.P.N. Nampoori, V. Ramakrishnan, M. Umadevi, C.P.G. Vallabhan, Nonlinear optical absorption in silver nanosol, J. Phys. Appl. Phys. 36 (2003) 1242-1245. doi:10.1088/0022-3727/36/11/303.

[65] A.M. Heikal, M.F.O. Hameed, S.S.A. Obayya, Basic Principles of Surface Plasmon Resonance, in: M.F.O. Hameed, S. Obayya (Eds.), Comput. Photonic Sens., Springer International Publishing, Cham, 2019: pp. 53-72. doi:10.1007/978-3-319-76556-3_3.

[66] A. Agrawal, S.H. Cho, O. Zandi, S. Ghosh, R.W. Johns, D.J. Milliron, Localized Surface Plasmon Resonance in Semiconductor Nanocrystals, Chem. Rev. 118 (2018) 3121-3207. doi:10.1021/acs.chemrev.7b00613.

[67] V.G. Kravets, A.V. Kabashin, W.L. Barnes, A.N. Grigorenko, Plasmonic Surface Lattice Resonances: A Review of Properties and Applications, Chem. Rev. 118 (2018) 5912-5951. doi:10.1021/acs.chemrev.8b00243.

[68] N.M. Gabor, J.C.W. Song, Q. Ma, N.L. Nair, T. Taychatanapat, K. Watanabe, T. Taniguchi, L.S. Levitov, P. Jarillo-Herrero, Hot Carrier-Assisted Intrinsic Photoresponse in Graphene, Science. 334 (2011) 648-652. doi:10.1126/science. 1211384 .

[69] S. Ghosh, S.M. Bachilo, R.A. Simonette, K.M. Beckingham, R.B. Weisman, Oxygen Doping Modifies Near-Infrared Band Gaps in Fluorescent Single-Walled Carbon Nanotubes, Science. 330 (2010) 1656-1659. doi:10.1126/science.1196382.

[70] K.J. Tielrooij, L. Piatkowski, M. Massicotte, A. Woessner, Q. Ma, Y. Lee, K.S. Myhro, C.N. Lau, P. Jarillo-Herrero, N.F. van Hulst, F.H.L. Koppens, Generation of photovoltage in graphene on a femtosecond timescale through efficient carrier heating, Nat. Nanotechnol. 10 (2015) 437-443. doi:10.1038/nnano.2015.54.

[71] K.J. Tielrooij, J.C.W. Song, S.A. Jensen, A. Centeno, A. Pesquera, A. Zurutuza Elorza, M. Bonn, L.S. Levitov, F.H.L. Koppens, Photoexcitation cascade and multiple hotcarrier generation in graphene, Nat. Phys. 9 (2013) 248-252. doi:10.1038/nphys2564.

[72] K.M. Lee, M.L. Smith, H. Koerner, N. Tabiryan, R.A. Vaia, T.J. Bunning, T.J. White, Photodriven, Flexural-Torsional Oscillation of Glassy Azobenzene Liquid Crystal Polymer Networks, Adv. Funct. Mater. 21 (2011) 2913-2918. doi:10.1002/adfm.201100333.

[73] H.K. Bisoyi, A.M. Urbas, Q. Li, Soft Materials Driven by Photothermal Effect and Their Applications, Adv. Opt. Mater. 6 (2018) 1800458. doi:10.1002/adom.201800458.

[74] L. Dong, Y. Zhao, Photothermally driven liquid crystal polymer actuators, Mater. Chem. Front. 2 (2018) 1932-1943. doi:10.1039/C8QM00363G. 
[75] X. Lu, H. Zhang, G. Fei, B. Yu, X. Tong, H. Xia, Y. Zhao, Liquid-Crystalline Dynamic Networks Doped with Gold Nanorods Showing Enhanced Photocontrol of Actuation, Adv. Mater. 30 (2018) 1706597. doi:10.1002/adma.201706597.

[76] W. Wei, Z. Zhang, J. Wei, X. Li, J. Guo, Phototriggered Selective Actuation and SelfOscillating in Dual-Phase Liquid Crystal Photonic Actuators, Adv. Opt. Mater. 6 (2018) 1800131. doi:10.1002/adom.201800131.

[77] Y. Yang, W. Zhan, R. Peng, C. He, X. Pang, D. Shi, T. Jiang, Z. Lin, GrapheneEnabled Superior and Tunable Photomechanical Actuation in Liquid Crystalline Elastomer Nanocomposites, Adv. Mater. $27 \quad$ (2015) 6376-6381. doi:10.1002/adma.201503680.

[78] L. Wang, H.K. Bisoyi, Z. Zheng, K.G. Gutierrez-Cuevas, G. Singh, S. Kumar, T.J. Bunning, Q. Li, Stimuli-directed self-organized chiral superstructures for adaptive windows enabled by mesogen-functionalized graphene, Mater. Today. 20 (2017) 230237. doi:10.1016/j.mattod.2017.04.028.

[79] C.J. Camargo, H. Campanella, J.E. Marshall, N. Torras, K. Zinoviev, E.M. Terentjev, J. Esteve, Localised Actuation in Composites Containing Carbon Nanotubes and Liquid Crystalline Elastomers, Macromol. Rapid Commun. 32 (2011) 1953-1959. doi:10.1002/marc.201100578.

[80] H.K.F. Cheng, T. Basu, N.G. Sahoo, L. Li, S.H. Chan, Current Advances in the Carbon Nanotube/Thermotropic Main-Chain Liquid Crystalline Polymer Nanocomposites and Their Blends, Polymers. 4 (2012) 889-912. doi:10.3390/polym4020889.

[81] L. Yang, K. Setyowati, A. Li, S. Gong, J. Chen, Reversible Infrared Actuation of Carbon Nanotube-Liquid Crystalline Elastomer Nanocomposites, Adv. Mater. 20 (2008) 2271-2275. doi:10.1002/adma.200702953.

[82] Y. Ji, Y.Y. Huang, R. Rungsawang, E.M. Terentjev, Dispersion and Alignment of Carbon Nanotubes in Liquid Crystalline Polymers and Elastomers, Adv. Mater. 22 (2010) 3436-3440. doi:10.1002/adma.200904103.

[83] J.E. Marshall, Y. Ji, N. Torras, K. Zinoviev, E.M. Terentjev, Carbon-nanotube sensitized nematic elastomer composites for IR-visible photo-actuation, Soft Matter. 8 (2012) 1570-1574. doi:10.1039/C1SM06656K.

[84] N. Torras, J.E. Marshall, K.E. Zinoviev, C.J. Camargo, E.M. Terentjev, J. Esteve, GasPressure Molding-Based Fabrication of Smart Actuators from Nematic LiquidCrystalline Elastomer: Gas-Pressure Molding-Based Fabrication of Smart LCE Actuators, Macromol. Mater. Eng. 299 (2014) 1163-1169. doi:10.1002/mame.201300462.

[85] Q. Chen, Y. Wei, Y. Ji, Photo-responsive liquid crystalline vitrimer containing oligoanilines, Chin. Chem. Lett. 28 (2017) 2139-2142. doi:10.1016/j.cclet.2017.09.011.

[86] Z. Li, Y. Yang, Z. Wang, X. Zhang, Q. Chen, X. Qian, N. Liu, Y. Wei, Y. Ji, Polydopamine nanoparticles doped in liquid crystal elastomers for producing dynamic 3D structures, J. Mater. Chem. A. 5 (2017) 6740-6746. doi:10.1039/C7TA00458C.

[87] Y. Yang, Z. Pei, Z. Li, Y. Wei, Y. Ji, Making and Remaking Dynamic 3D Structures by Shining Light on Flat Liquid Crystalline Vitrimer Films without a Mold, J. Am. Chem. Soc. 138 (2016) 2118-2121. doi:10.1021/jacs.5b12531.

[88] Z. Pei, Y. Yang, Q. Chen, E.M. Terentjev, Y. Wei, Y. Ji, Mouldable liquid-crystalline elastomer actuators with exchangeable covalent bonds, Nat. Mater. 13 (2014) 36-41. doi:10.1038/nmat3812. 
[89] S. Fu, H. Zhang, Y. Zhao, Optically and thermally activated shape memory supramolecular liquid crystalline polymers, J. Mater. Chem. C. 4 (2016) 4946-4953. doi:10.1039/C6TC00718J.

[90] L. Liu, M.-H. Liu, L.-L. Deng, B.-P. Lin, H. Yang, Near-Infrared Chromophore Functionalized Soft Actuator with Ultrafast Photoresponsive Speed and Superior Mechanical Property, J. Am. Chem. Soc. 139 (2017) 11333-11336. doi:10.1021/jacs.7b06410.

[91] O. Köysal, M. Okutan, M. Gökçen, Investigation of dielectric properties and diffraction efficiency enhancements caused by photothermal effect in DR9 dye-doped nematic liquid crystal, Opt. Commun. $284 \quad$ (2011) 4924-4928. doi:10.1016/j.optcom.2011.06.046.

[92] H. Ono, Y. Harato, N. Kawatsuki, Photothermal effects in liquid crystals on dye-doped polymer films, J. Appl. Phys. 83 (1998) 4957-4962. doi:10.1063/1.367297.

[93] H. Bian, F. Yao, H. Liu, F. Huang, Y. Pei, C. Hou, X. Sun, Optically controlled random lasing based on photothermal effect in dye-doped nematic liquid crystals, Liq. Cryst. 41 (2014) 1436-1441. doi:10.1080/02678292.2014.924162.

[94] W. Liu, L.-X. Guo, B.-P. Lin, X.-Q. Zhang, Y. Sun, H. Yang, Near-Infrared Responsive Liquid Crystalline Elastomers Containing Photothermal Conjugated Polymers, Macromolecules. 49 (2016) 4023-4030. doi:10.1021/acs.macromol.6b00640.

[95] S. Bongiovanni Abel, M.A. Molina, C.R. Rivarola, M.J. Kogan, C.A. Barbero, Smart polyaniline nanoparticles with thermal and photothermal sensitivity, Nanotechnology. 25 (2014) 495602. doi:10.1088/0957-4484/25/49/495602.

[96] K. Brach, J. Olesiak-Banska, M. Waszkielewicz, M. Samoc, K. Matczyszyn, DNA liquid crystals doped with AuAg nanoclusters: One-photon and two-photon imaging, J. Mol. Liq. 259 (2018) 82-87. doi:10.1016/j.molliq.2018.02.108.

[97] L. Pezzi, L. De Sio, A. Veltri, T. Placido, G. Palermo, R. Comparelli, M.L. Curri, A. Agostiano, N. Tabiryan, C. Umeton, Photo-thermal effects in gold nanoparticles dispersed in thermotropic nematic liquid crystals, Phys. Chem. Chem. Phys. 17 (2015) 20281-20287. doi:10.1039/C5CP01377A.

[98] M.M. Wójcik, J. Wróbel, Z.Z. Jańczuk, J. Mieczkowski, E. Górecka, J. Choi, M. Cho, D. Pociecha, Liquid-Crystalline Elastomers with Gold Nanoparticle Cross-Linkers, Chem. - Eur. J. 23 (2017) 8912-8920. doi:10.1002/chem.201700723.

[99] X. Liu, R. Wei, P.T. Hoang, X. Wang, T. Liu, P. Keller, Reversible and Rapid Laser Actuation of Liquid Crystalline Elastomer Micropillars with Inclusion of Gold Nanoparticles, Adv. Funct. Mater. 25 (2015) 3022-3032. doi:10.1002/adfm.201500443.

[100] K.G. Gutierrez-Cuevas, L. Wang, C. Xue, G. Singh, S. Kumar, A. Urbas, Q. Li, Near infrared light-driven liquid crystal phase transition enabled by hydrophobic mesogen grafted plasmonic gold nanorods, Chem. Commun. 51 (2015) 9845-9848. doi:10.1039/C5CC02127H.

[101] Z.-Y. Kuang, Y.-J. Fan, L. Tao, M.-L. Li, N. Zhao, P. Wang, E.-Q. Chen, F. Fan, H.-L. Xie, Alignment Control of Nematic Liquid Crystal using Gold Nanoparticles Grafted by the Liquid Crystalline Polymer with Azobenzene Mesogens as the Side Chains, ACS Appl. Mater. Interfaces. 10 (2018) 27269-27277. doi:10.1021/acsami.8b07483.

[102] T. Yamamoto, J. Noguchi, M. Kinoshita, Y. Takenaka, H. Kihara, Near-infrared-lightresponsive liquid-crystalline composite gels with photo-healing ability, Mol. Cryst. Liq. Cryst. 662 (2018) 38-45. doi:10.1080/15421406.2018.1466239. 
[103] J. Gao, Y. Sun, J. Zhou, Z. Zheng, H. Chen, W. Su, Q. Zhang, Preparation of Ag nanoparticles termini-protected side-chain liquid crystalline azobenzene polymers by RAFT polymerization, J. Polym. Sci. Part Polym. Chem. 45 (2007) 5380-5386. doi:10.1002/pola.22282.

[104] J. Zhang, J. Wang, L. Zhao, W. Yang, M. Bi, Y. Wang, H. Niu, Y. Li, B. Wang, Y. Gao, C. Li, X. Huang, Photo responsive silver nanoparticles incorporated liquid crystalline elastomer nanocomposites based on surface plasmon resonance, Chem. Res. Chin. Univ. 33 (2017) 839-846. doi:10.1007/s40242-017-7067-0.

[105] M. Ciancone, F. Camerel, Laser triggered phase transition in photothermal liquid crystals, Chem. Commun. 53 (2017) 6339-6342. doi:10.1039/C7CC03318D.

[106] I. Bahnini, M. Rivette, A. Rechia, A. Siadat, A. Elmesbahi, Additive manufacturing technology: the status, applications, and prospects, Int. J. Adv. Manuf. Technol. 97 (2018) 147-161. doi:10.1007/s00170-018-1932-y.

[107] N. Deoray, B. Kandasubramanian, Review on Three-Dimensionally Emulated FiberEmbedded Lactic Acid Polymer Composites: Opportunities in Engineering Sector, Polym.-Plast. Technol. Eng. 57 (2018) 860-874. doi:10.1080/03602559.2017.1354226.

[108] M. Zarek, M. Layani, S. Eliazar, N. Mansour, I. Cooperstein, E. Shukrun, A. Szlar, D. Cohn, S. Magdassi, 4D printing shape memory polymers for dynamic jewellery and fashionwear, Virtual Phys. Prototyp. $11 \quad$ (2016) 263-270. doi:10.1080/17452759.2016.1244085.

[109] M.K. Hausmann, P.A. Rühs, G. Siqueira, J. Läuger, R. Libanori, T. Zimmermann, A.R. Studart, Dynamics of Cellulose Nanocrystal Alignment during 3D Printing, ACS Nano. 12 (2018) 6926-6937. doi:10.1021/acsnano.8b02366.

[110] J.M. Korde, M. Shaikh, B. Kandasubramanian, Bionic Prototyping of Honeycomb Patterned Polymer Composite and Its Engineering Application, Polym.-Plast. Technol. Eng. (2018) 1-17. doi:10.1080/03602559.2018.1434667.

[111] R. Yadav, M. Naebe, X. Wang, B. Kandasubramanian, Review on 3D Prototyping of Damage Tolerant Interdigitating Brick Arrays of Nacre, Ind. Eng. Chem. Res. 56 (2017) 10516-10525. doi:10.1021/acs.iecr.7b01679.

[112] S. Gharde, A. Surendren, J.M. Korde, S. Saini, N. Deoray, R. Goud, S. Nimje, B. Kandasubramanian, Recent Advances in Additive Manufacturing of Bio-inspired Materials, in: C. Prakash, S. Singh, R. Singh, S. Ramakrishna, B.S. Pabla, S. Puri, M.S. Uddin (Eds.), Biomanufacturing, Springer International Publishing, Cham, 2019: pp. 35-68. doi:10.1007/978-3-030-13951-3_2.

[113] C.M. González-Henríquez, M.A. Sarabia-Vallejos, J. Rodriguez-Hernandez, Polymers for additive manufacturing and 4D-printing: Materials, methodologies, and biomedical applications, Prog. Polym. Sci. (2019) S007967001830128X. doi:10.1016/j.progpolymsci.2019.03.001.

[114] J. Gardan, Smart materials in additive manufacturing: state of the art and trends, Virtual Phys. Prototyp. 14 (2019) 1-18. doi:10.1080/17452759.2018.1518016.

[115] A. Prasad, B. Kandasubramanian, Fused deposition processing polycaprolactone of composites for biomedical applications, Polym.-Plast. Technol. Mater. (2019) 1-34. doi:10.1080/25740881.2018.1563117.

[116] Y.S. Lui, W.T. Sow, L.P. Tan, Y. Wu, Y. Lai, H. Li, 4D Printing and Stimuliresponsive Materials in Biomedical Applications, Acta Biomater. (2019) S1742706119303204. doi:10.1016/j.actbio.2019.05.005. 
[117] P. Rastogi, B. Kandasubramanian, Breakthrough in the printing tactics for stimuliresponsive materials: 4D printing, Chem. Eng. J. 366 (2019) 264-304. doi:10.1016/j.cej.2019.02.085.

[118] T.D. Ngo, A. Kashani, G. Imbalzano, K.T.Q. Nguyen, D. Hui, Additive manufacturing (3D printing): A review of materials, methods, applications and challenges, Compos. Part B Eng. 143 (2018) 172-196. doi:10.1016/j.compositesb.2018.02.012.

[119] A. Güney, J. Malda, W.J.A. Dhert, D.W. Grijpma, Triblock copolymers based on $\varepsilon-$ caprolactone and trimethylene carbonate for the 3D printing of tissue engineering scaffolds, Int. J. Artif. Organs. 40 (2017) 176-184. doi:10.5301/ijao.5000543.

[120] A. Kirillova, R. Maxson, G. Stoychev, C.T. Gomillion, L. Ionov, 4D Biofabrication Using Shape-Morphing Hydrogels, Adv. Mater. $29 \quad$ (2017) 1703443. doi:10.1002/adma.201703443.

[121] L. Roseti, C. Cavallo, G. Desando, V. Parisi, M. Petretta, I. Bartolotti, B. Grigolo, Three-Dimensional Bioprinting of Cartilage by the Use of Stem Cells: A Strategy to Improve Regeneration, Materials. 11 (2018) 1749. doi:10.3390/ma11091749.

[122] S. Jasveer, X. Jianbin, Comparison of Different Types of 3D Printing Technologies, Int. J. Sci. Res. Publ. IJSRP. 8 (2018). doi:10.29322/IJSRP.8.4.2018.p7602.

[123] O. Guillaume, M.A. Geven, C.M. Sprecher, V.A. Stadelmann, D.W. Grijpma, T.T. Tang, L. Qin, Y. Lai, M. Alini, J.D. de Bruijn, H. Yuan, R.G. Richards, D. Eglin, Surface-enrichment with hydroxyapatite nanoparticles in stereolithography-fabricated composite polymer scaffolds promotes bone repair, Acta Biomater. 54 (2017) 386-398. doi:10.1016/j.actbio.2017.03.006.

[124] W. Gao, Y. Zhang, D. Ramanujan, K. Ramani, Y. Chen, C.B. Williams, C.C.L. Wang, Y.C. Shin, S. Zhang, P.D. Zavattieri, The status, challenges, and future of additive manufacturing in engineering, Comput.-Aided Des. 69 (2015) 65-89. doi:10.1016/j.cad.2015.04.001.

[125] sandeep, deepak chhabra, Comparison and analysis of different $3 \mathrm{~d}$ printing techniques, Int. J. Latest Trends Eng. Technol. 8 (2017). doi:10.21172/1.841.44.

[126] N. Mishra, B. Kandasubramanian, Biomimetic Design of Artificial Materials Inspired by Iridescent Nacre Structure and Its Growth Mechanism, Polym.-Plast. Technol. Eng. (2017) 1-15. doi:10.1080/03602559.2017.1326139.

[127] R. Yadav, R. Goud, A. Dutta, X. Wang, M. Naebe, B. Kandasubramanian, Biomimicking of Hierarchal Molluscan Shell Structure Via Layer by Layer 3D Printing, Ind. Eng. Chem. Res. 57 (2018) 10832-10840. doi:10.1021/acs.iecr.8b01738.

[128] J. ten Kate, G. Smit, P. Breedveld, 3D-printed upper limb prostheses: a review, Disabil. Rehabil. Assist. Technol. 12 (2017) 300-314. doi:10.1080/17483107.2016.1253117.

[129] R.S. Ambekar, B. Kandasubramanian, Progress in the Advancement of Porous Biopolymer Scaffold: Tissue Engineering Application, Ind. Eng. Chem. Res. 58 (2019) 6163-6194. doi:10.1021/acs.iecr.8b05334.

[130] A. Malik, B. Kandasubramanian, Flexible Polymeric Substrates for Electronic Applications, Polym. Rev. (2018) 1-38. doi:10.1080/15583724.2018.1473424.

[131] M. Li, Q. Yang, H. Liu, M. Qiu, T.J. Lu, F. Xu, Capillary Origami Inspired Fabrication of Complex 3D Hydrogel Constructs, Small. 12 (2016) 4492-4500. doi:10.1002/smll.201601147.

[132] Y.L. Yap, W.Y. Yeong, Additive manufacture of fashion and jewellery products: a mini review: This paper provides an insight into the future of $3 \mathrm{D}$ printing industries for 
fashion and jewellery products, Virtual Phys. Prototyp. 9 (2014) 195-201. doi:10.1080/17452759.2014.938993.

[133] S. Tibbits, C. McKnelly, C. Olguin, D. Dikovsky, S. Hirsch, 4d Printing And Universal Transformation, Material Agency, Mater. Agency. (2014) 10.

[134] H. Xie, K.-K. Yang, Y.-Z. Wang, Photo-cross-linking: A powerful and versatile strategy to develop shape-memory polymers, Prog. Polym. Sci. 95 (2019) 32-64. doi:10.1016/j.progpolymsci.2019.05.001.

[135] C.P. Ambulo, J.J. Burroughs, J.M. Boothby, H. Kim, M.R. Shankar, T.H. Ware, Fourdimensional Printing of Liquid Crystal Elastomers, ACS Appl. Mater. Interfaces. 9 (2017) 37332-37339. doi:10.1021/acsami.7b11851.

[136] Joanne Teoh, Jia An, Xiaofan Feng, Yue Zhao, Chee Chua, Yong Liu, Design and 4D Printing of Cross-Folded Origami Structures: A Preliminary Investigation, Materials. 11 (2018) 376. doi:10.3390/ma11030376.

[137] M. Kang, Y. Pyo, J. young Jang, Y. Park, Y.-H. Son, M. Choi, J. wan Ha, Y.-W. Chang, C.S. Lee, Design of a shape memory composite(SMC) using 4D printing technology, Sens. Actuators Phys. 283 (2018) 187-195. doi:10.1016/j.sna.2018.08.049.

[138] G. Liu, Y. Zhao, G. Wu, J. Lu, Origami and 4D printing of elastomer-derived ceramic structures, Sci. Adv. (2018) 11.

[139] M.C. Mulakkal, R.S. Trask, V.P. Ting, A.M. Seddon, Responsive cellulose-hydrogel composite ink for 4D printing, Mater. Des. 160 (2018) 108-118. doi:10.1016/j.matdes.2018.09.009.

[140] P. Zhu, W. Yang, R. Wang, S. Gao, B. Li, Q. Li, 4D Printing of Complex Structures with a Fast Response Time to Magnetic Stimulus, ACS Appl. Mater. Interfaces. 10 (2018) 36435-36442. doi:10.1021/acsami.8b12853.

[141] J.M. Korde, B. Kandasubramanian, Fundamentals and Effects of Biomimicking Stimuli-Responsive Polymers for Engineering Functions, Ind. Eng. Chem. Res. (2019) acs.iecr.9b00683. doi:10.1021/acs.iecr.9b00683.

[142] X. Kuang, D.J. Roach, J. Wu, C.M. Hamel, Z. Ding, T. Wang, M.L. Dunn, H.J. Qi, Advances in 4D Printing: Materials and Applications, Adv. Funct. Mater. 29 (2019) 1805290. doi:10.1002/adfm.201805290.

[143] X. Li, J. Shang, Z. Wang, Intelligent materials: a review of applications in 4D printing, Assem. Autom. 37 (2017) 170-185. doi:10.1108/AA-11-2015-093.

[144] A.D. Auguste, J.W. Ward, J.O. Hardin, B.A. Kowalski, T.C. Guin, J.D. Berrigan, T.J. White, Enabling and Localizing Omnidirectional Nonlinear Deformation in Liquid Crystalline Elastomers, Adv. Mater. 30 (2018) 1802438. doi:10.1002/adma.201802438.

[145] C. Yuan, D.J. Roach, C.K. Dunn, Q. Mu, X. Kuang, C.M. Yakacki, T.J. Wang, K. Yu, H.J. Qi, 3D printed reversible shape changing soft actuators assisted by liquid crystal elastomers, Soft Matter. 13 (2017) 5558-5568. doi:10.1039/C7SM00759K.

[146] D.J. Roach, X. Kuang, C. Yuan, K. Chen, H.J. Qi, Novel ink for ambient condition printing of liquid crystal elastomers for 4D printing, Smart Mater. Struct. 27 (2018) 125011. doi:10.1088/1361-665X/aae96f.

[147] B.A. Kowalski, T.C. Guin, A.D. Auguste, N.P. Godman, T.J. White, Pixelated Polymers: Directed Self Assembly of Liquid Crystalline Polymer Networks, ACS Macro Lett. 6 (2017) 436-441. doi:10.1021/acsmacrolett.7b00116.

[148] S. Shabana, R. Prasansha, I. Kalinina, I. Potoroko, U. Bagale, S.H. Shirish, Ultrasound assisted acid hydrolyzed structure modification and loading of antioxidants on potato 
starch nanoparticles, Ultrason. Sonochem. $51 \quad$ (2019) 444-450. doi:10.1016/j.ultsonch.2018.07.023.

[149] X. Huang, J. He, K. Sun, Y. Chen, Z. Zha, C. Zhou, Liquid crystal behavior and cytocompatibility of graphene oxide dispersed in sodium alginate solutions, Carbon. 129 (2018) 258-269. doi:10.1016/j.carbon.2017.12.006.

[150] D.C. Yeo, E.R. Balmayor, J.-T. Schantz, C. Xu, Microneedle physical contact as a therapeutic for abnormal scars, Eur. J. Med. Res. 22 (2017). doi:10.1186/s40001-0170269-6.

[151] O. Dmitrenko, T. Orlova, I. Terenetskaya, Medium controlled photochemistry of Provitamin D: From solutions to liquid crystals, J. Mol. Liq. 267 (2018) 428-435. doi:10.1016/j.molliq.2018.01.049.

[152] Q. He, Z. Wang, Z. Song, S. Cai, Bioinspired Design of Vascular Artificial Muscle, Adv. Mater. Technol. (2018) 1800244. doi:10.1002/admt.201800244.

[153] A.H. Gelebart, G. Vantomme, E.W. Meijer, D.J. Broer, Mastering the Photothermal Effect in Liquid Crystal Networks: A General Approach for Self-Sustained Mechanical Oscillators, Adv. Mater. 29 (2017) 1606712. doi:10.1002/adma.201606712.

[154] S.W. Ula, N.A. Traugutt, R.H. Volpe, R.R. Patel, K. Yu, C.M. Yakacki, Liquid crystal elastomers: an introduction and review of emerging technologies, Liq. Cryst. Rev. 6 (2018) 78-107. doi:10.1080/21680396.2018.1530155.

[155] MarketsandMarkets, 4D Printing Market worth \$537.8 Million by 2025, (2015). https://www.marketsandmarkets.com/PressReleases/4d-printing.asp (accessed August 20, 2018). 


\section{Highlights}

- Discussion of liquid crystals undergoing photothermal response

- Outline on agents as heat-engendering source in electromagnetic radiation

- Prospect of photothermal actuation in liquid crystals with 4D Printing

- Exploration of liquid crystals in biomedical treatment 